\title{
Statistical Properties of Radiation from VUV and X-ray Free Electron Laser
}

\author{
E.L. Saldin ${ }^{\text {a }}$, E.A. Schneidmiller ${ }^{a}$ and M.V. Yurkov ${ }^{b}$ \\ a Automatic Systems Corporation, 443050 Samara, Russia \\ ${ }^{\mathrm{b}}$ Joint Institute for Nuclear Research, Dubna, 141980 Moscow Region, Russia
}

\begin{abstract}
The paper presents comprehensive analysis of statistical properties of the radiation from self amplified spontaneous emission (SASE) free electron laser operating in linear and nonlinear mode. The investigation has been performed in a one-dimensional approximation assuming the electron pulse length to be much larger than a coherence length of the radiation. The following statistical properties of the SASE FEL radiation have been studied in detail: time and spectral field correlations, distribution of the fluctuations of the instant radiation power, distribution of the energy in the electron bunch, distribution of the radiation energy after monochromator installed at the FEL amplifier exit and the radiation spectrum. Linear high gain limit is studied analytically. It is shown that the radiation from SASE FEL operating in linear regime possesses all the features corresponding to completely chaotic polarized radiation. Detailed study of statistical properties of the radiation from SASE FEL operating in linear and nonlinear regime has been performed by means of time-dependent simulation codes.

All numerical results presented in the paper have been calculated for the $70 \mathrm{~nm}$ SASE FEL at the TESLA Test Facility being under construction at DESY.
\end{abstract}




\section{Introduction}

Self amplified spontaneous emission free electron laser (SASE FEL) $[1,2,3,4]$ is considered now as perspective source of coherent VUV and X-ray radiation. The projects of X-ray SASE FELs are developed at SLAC and DESY [5, 6] and $6 \mathrm{~nm}$ SASE FEL is under construction at the TESLA Test Facility (TTF) at DESY $[7,8]$. The first phase of the TTF FEL project will operate at the radiation wavelength $40-100 \mathrm{~nm}$ [9]. Peak brilliance of the SASE FEL radiation will exceed by 6 - 10 orders of magnitude the corresponding value available at the third generation synchrotron radiation sources. That is why the plans of future experiments with the SASE FEL radiation are intensively discussed nowadays [10].

Correct design of the SASE FEL and planning of user's equipment and experiments depend strongly on knowledge of the radiation amplification process in the SASE FEL and properties of the output radiation. The SASE FEL is a single pass FEL amplifier without external seeding. The process of amplification in the SASE FEL starts from the shot noise in the electron beam having stochastic nature. It means that the SASE FEL radiation is also stochastic object, that is why there exists definite problem for description of the SASE FEL process requiring development of time-dependent theory of the FEL amplifier. Till now there is no comprehensive theoretical results describing SASE FEL process in detail. Some averaged output characteristics of SASE FEL have been obtained in refs. $[1,2,3,12,13,14,15,16]$. Quantum consideration of photon statistics in SASE FEL has been performed in ref. [11]. An approach for time-dependent numerical simulations of SASE FEL radiation has been developed in ref. [16]. Realization of this approach allowed to obtain some statistical properties of the radiation from SASE FEL operating in linear and nonlinear regime $[16,17]$.

Nevertheless, the previous studies do not give comprehensive description of the output radiation from SASE FEL and the following statistical properties should studied in detail:

- Time and spectral field correlations;

- Statistics of the instantaneous radiation power;

- Statistics of the finite-time integrals of the instantaneous power;

- Statistics of the radiation energy after the monochromator installed at the exit of SASE FEL;

- The shape of the radiation spectrum;

- Photoelectric counting statistics of SASE FEL radiation.

This paper gives comprehensive answers on all the above mentioned problems describing statistical properties of the radiation from SASE FEL operating in linear and nonlinear 
regime. The investigation has been performed in a one-dimensional approximation. Practically important case is considered when the electron pulse length is much larger than a coherence length of the radiation. Linear high gain limit is described analytically. Analytical formulae for the first and the second order time and spectral correlation functions have been obtained. It is shown that the radiation from SASE FEL operating in the linear regime possesses all the features corresponding to completely chaotic polarized radiation. In particular, the higher order correlation functions (time and spectral) are expressed via the first order correlation function, the probability density distribution of the instant radiation power follows the negative exponential distribution and the probability density function of the finite-time integrals of the instantaneous power and of the energy after monochromator follows the gamma distribution.

More detailed analysis of the SASE FEL operation is performed by linear and nonlinear time-dependent simulation codes. These codes have been constructed on the base of existent steady-state 1-D code package FS1D [21] using time-dependent technique developed in ref. [16]. For precise reconstruction of the radiation spectrum a special technique has been developed using Fourier transformation of the first order time correlation function. Using statistical analysis of a large number of runs we calculated numerically all characteristics of the radiation from SASE FEL operating in linear and nonlinear regime.

Evolution of the radiation spectrum has been traced from the beginning of the amplification process up to deep nonlinear regime. In particular, it is demonstrated that at the beginning of the SASE FEL process the spectrum shape exactly corresponds to the spectrum of the undulator radiation. Averaged spectrum of the radiation from the SASE FEL operating in the nonlinear regime has been calculated for the first time. It has been shown also that statistics of the radiation from SASE FEL operating at saturation changes significantly with respect to the linear mode of operation.

The paper is organized as follows. Statistical properties of the shot noise in the electron beam and analytical treatment of the high-gain linear mode of SASE FEL operation is presented in section 2. The photoelectric detection of the SASE FEL radiation is discussed in section 3 . The time-dependent simulation algorithms are described in section 4 and the results of numerical study of the SASE FEL process are presented in section 5 .

To be specific, we calculated all the numerical results for the $70 \mathrm{~nm}$ SASE FEL at the TESLA Test Facility being under construction at DESY [9]. Nevertheless, they can be simply scaled for calculation of another SASE FELs by means of application of similarity techniques (see, e.g. ref. $[20,21]$ ). 


\section{Analytical treatment of statistical properties of SASE FEL radiation in a high-gain linear regime}

In the linear mode of operation the SASE FEL can be treated as a narrow band linear device which filters a wide band random input signal - shot noise. General property of such devices is that an output signal is a Gaussian random process. In other words, the output signal is composed of a multitude of independent additive contributions and, according to the central limit theorem, this leads to Gaussian statistics. It does not mean that any considered physical variable is distributed in accordance with the Gaussian probability density function. For instance, in the case of SASE FEL the real and imaginary parts of slowly varying complex amplitudes of electric field of electromagnetic wave at the FEL amplifier exit have Gaussian distribution. On the other hand, the instantaneous power, the finite-time integrals of the instantaneous power and the spectral density of the radiation energy have the other distributions as it will be shown below. Since electron pulse at the entrance of SASE FEL has finite duration, we deal with nonstationary random process. Analytical study of such a process in general case is very complicated, because short electron bunch combines the features of input signal and "active medium" with timedependent parameters. In other words, SASE FEL is a parametric amplifier. Analysis of design parameters of VUV and X-ray SASE FELs $[5,6,7,8,9]$ shows that the feature of these devices is that the bunch length is much larger than the radiation coherence length. This fact allows one to use such a model which provides the possibility of analytical description without lost of essential information about features of nonstationary process (correlations in spectrum, etc). Approximations satisfying these conditions are the using of rectangular profile of the electron bunch and application of the steady-state spectral Green's function. In the frame of this model it becomes possible to describe analytically all statistical properties of the radiation from SASE FEL operating in the high gain linear regime.

\subsection{Shot noise in the electron beam}

Fluctuations of the electron beam current density serve as input signal in SASE FEL. These fluctuations always exist in the electron beam due to the effect of shot noise. When the electron beam enters the undulator, the presence of the beam modulation at frequencies close to the resonance frequency of the FEL amplifier initiates the process of the amplification of coherent radiation. The shot noise in the electron beam has statistical nature which significantly influences on characteristics of the output radiation from SASE FEL. In this section we study statistical properties of the shot noise in the electron beam. 
Let us consider microscopic picture of the electron beam current at the entrance into the undulator. The electron beam current is constituted by moving electrons randomly arriving to the entrance of the undulator:

$$
I(t)=(-e) \sum_{k=1}^{N} \delta\left(t-t_{k}\right)
$$

where $\delta(\ldots)$ is delta-function, (-e) is the charge of the electron, $N$ is the number of electrons in a bunch and $t_{k}$ is random arrival time of the electron to the undulator entrance. The electron bunch profile is described by the profile function $F(t)$ and the beam current averaged over an ensemble of bunches can be written in the form:

$$
<I(t)>=(-e) N F(t) \text {. }
$$

The probability of arrival of the electron at time interval $t, t+d t$ is equal to $F(t) d t$. For the Gaussian profile of the electron bunch function $F(t)$ is of the form:

$$
F(t)=\frac{1}{\sqrt{2 \pi} \sigma_{T}} \exp \left(-\frac{t^{2}}{2 \sigma_{T}^{2}}\right)
$$

The electron beam current $I(t)$ and its Fourier transform $\bar{I}(\omega)$ are connected by Fourier transformations :

$$
\begin{aligned}
\bar{I}(\omega) & =\int_{-\infty}^{\infty} e^{i \omega t} I(t) d t=(-e) \sum_{k=1}^{N} e^{i \omega t_{k}}, \\
I(t) & =\frac{1}{2 \pi} \int_{-\infty}^{\infty} \bar{I}(\omega) e^{-i \omega t} d \omega=(-e) \sum_{k=1}^{N} \delta\left(t-t_{k}\right) .
\end{aligned}
$$

It follows from eq. (2.3) that the Fourier transform of the input current, $\bar{I}(\omega)$, is the sum of large number of complex phasors with random phases $\phi_{k}=\omega t_{k}$. If the characteristic duration of a bunch $\sigma_{T}$ is long, $\omega \sigma_{T} \gg 1$, then the phases $\phi_{k}$ can be considered to be uniformly distributed on interval $(-\pi, \pi)$. Under this condition the probability density distribution of $|\bar{I}(\omega)|^{2}$ is given by the negative exponential distribution [19]:

$$
p\left(|\bar{I}(\omega)|^{2}\right)=\frac{1}{\left\langle|\bar{I}(\omega)|^{2}\right\rangle} \exp \left(-\frac{|\bar{I}(\omega)|^{2}}{\left\langle|\bar{I}(\omega)|^{2}\right\rangle}\right),
$$

where $\langle\ldots\rangle$ means the averaging over ensemble of bunches.

Spectral correlation of the first order. Let us calculate the first order correlation of complex Fourier harmonics $\bar{I}(\omega)$ and $\bar{I}\left(\omega^{\prime}\right)$ :

$$
<\bar{I}(\omega) \bar{I}^{*}\left(\omega^{\prime}\right)>=e^{2}<\sum_{k=1}^{N} \sum_{n=1}^{N} \exp \left(i \omega t_{k}-i \omega^{\prime} t_{n}\right)>.
$$

Expanding this relation, we can write: 


$$
\begin{gathered}
<\bar{I}(\omega) \bar{I}^{*}\left(\omega^{\prime}\right)>=e^{2}<\sum_{k=1}^{N} \exp \left[i\left(\omega-\omega^{\prime}\right) t_{k}\right]>+e^{2}<\sum_{k \neq n} \exp \left(i \omega t_{k}-i \omega^{\prime} t_{n}\right)> \\
=e^{2} \sum_{k=1}^{N}<\exp \left[i\left(\omega-\omega^{\prime}\right) t_{k}\right]>+e^{2} \sum_{k \neq n}<\exp \left(i \omega t_{k}\right)><\exp \left(-i \omega^{\prime} t_{n}\right)>.
\end{gathered}
$$

Taking into account relation (2.1) and the first relation in eq. (2.3) we obtain that $<\exp \left(i \omega t_{k}\right)>$ is equal to the Fourier transform of the bunch profile function $F(t)$ :

$$
<\exp \left(i \omega t_{k}\right)>=\int_{-\infty}^{\infty} F\left(t_{k}\right) e^{i \omega t_{k}} d t_{k}=\bar{F}(\omega),
$$

Fourier transform of the Gaussian profile function (2.2) has the form:

$$
\bar{F}(\omega)=\exp \left(-\frac{\omega^{2} \sigma_{T}^{2}}{2}\right)
$$

Substituting eq. (2.6) into eq. (2.5), we obtain:

$$
<\bar{I}(\omega) \bar{I}^{*}\left(\omega^{\prime}\right)>=e^{2} N \bar{F}\left(\omega-\omega^{\prime}\right)+e^{2} N(N-1) \bar{F}(\omega) \bar{F}^{*}\left(\omega^{\prime}\right)
$$

In the case when

$$
N|\tilde{F}(\omega)|^{2} \ll 1
$$

we can write the following expression for the first order correlation:

$$
<\bar{I}(\omega) \bar{I}^{*}\left(\omega^{\prime}\right)>=e^{2} N \bar{F}\left(\omega-\omega^{\prime}\right)
$$

For specific cases of the Gaussian and rectangular profile of the electron bunch the first order correlation of complex Fourier harmonics $\bar{I}(\omega)$ and $\bar{I}\left(\omega^{\prime}\right)$ has the form:

Gaussian profile:

$$
<\bar{I}(\omega) \bar{I}^{*}\left(\omega^{\prime}\right)>=e^{2} N \exp \left[-\frac{\left(\omega-\omega^{\prime}\right)^{2} \sigma_{T}^{2}}{2}\right],
$$

rectangular profile:

$$
<\bar{I}(\omega) \bar{I}^{*}\left(\omega^{\prime}\right)>=e^{2} N \frac{\sin \frac{\left(\omega-\omega^{\prime}\right) T}{2}}{\frac{\left(\omega-\omega^{\prime}\right) T}{2}},
$$

where $T$ is the pulse duration of rectangular electron bunch.

Let us discuss practical applicability region of approximation (2.7). Physical sense of relation (2.7) is that at given pulse duration $\sigma_{T}$, frequency $\omega$ has to be large enough $\left(\sigma_{T} \omega \gg 1\right)$. Let us consider specific numerical example for the case of Gaussian profile of the bunch. At $\sigma_{T} \omega=10$ we have $|\bar{F}(\omega)|^{2}=\exp (-100)$. As a rule, the number of the particles the bunch $N$ is not larger than $10^{11}$, so condition (2.7) is fulfilled in practice. 
It is necessary to give some comments on the case of rectangular bunch. Formally in this case the applicability region of condition (2.7) is less than for the case of the Gaussian bunch of the same duration. This is due to the fact that the bunch form factor $|\bar{F}(\omega)|^{2}$ decreases slowly with the frequency increase. On the other hand, in a realistic situation there is no rigid boundary of the bunch and the beam current falls to zero value during some time interval $\Delta \sigma_{T}, \Delta \sigma_{T} \ll T$. When the beam current at the edge falls in accordance with the Gaussian law, $\Delta \sigma_{T}$ must obey the following conditions:

$$
\frac{\Delta \sigma_{T}}{T} \ll 1, \quad \Delta \sigma_{T} \omega \gg 1, \quad \frac{N}{\left(\Delta \sigma_{T} \omega\right)^{4}(\omega T)^{2}} \ll 1 .
$$

In this case expression $(2.9 \mathrm{~b})$ is valid within the boundaries:

$$
\left|\left(\omega-\omega^{\prime}\right)\right| \ll \frac{1}{\Delta \sigma_{T}} .
$$

Spectral correlation of the second order. Let us calculate the second order correlation of complex Fourier harmonics $\bar{I}(\omega)$ and $\bar{I}\left(\omega^{\prime}\right)$ :

$$
<|\bar{I}(\omega)|^{2}\left|\bar{I}\left(\omega^{\prime}\right)\right|^{2}>=e^{4}<\sum_{n=1}^{N} \sum_{m=1}^{N} \sum_{p=1}^{N} \sum_{q=1}^{N} \exp \left[i \omega\left(t_{n}-t_{m}\right)+i \omega^{\prime}\left(t_{p}-t_{q}\right)\right]>.
$$

The $N^{4}$ terms in this summation can be placed in 15 different classes (see, for instance, ref. [19]). In the case when condition (2.7) is fulfilled, only two classes are of importance with $(n=m, p=q, n \neq p)$ and $(n=q, m=p, n \neq m)$. Thus, we can write:

$$
<|\bar{I}(\omega)|^{2}\left|\bar{I}\left(\omega^{\prime}\right)\right|^{2}>=<|\bar{I}(\omega)|^{2}><\left|\bar{I}\left(\omega^{\prime}\right)\right|^{2}>+\left|<\bar{I}(\omega) \bar{I}^{*}\left(\omega^{\prime}\right)>\right|^{2} .
$$

Substituting eq. (2.8) into eq. (2.10) we obtain:

$$
<|\bar{I}(\omega)|^{2}\left|\bar{I}\left(\omega^{\prime}\right)\right|^{2}>=e^{4} N^{2}\left(1+\left|\bar{F}\left(\omega-\omega^{\prime}\right)\right|^{2}\right) .
$$

\subsection{Green's function}

In the framework of the one-dimensional model and when the effects of the space charge field and energy spread in the beam can be neglected, operation of the FEL amplifier is described in terms of the gain parameter $\Gamma$ and the efficiency parameter $\rho$ (see, e.g. refs. $[3,20,21])$ :

$$
\Gamma=\left[\frac{2 \pi^{2} j_{0} K^{2} A_{J J}^{2}}{I_{A} \lambda_{\mathrm{w}} \gamma^{3}}\right]^{1 / 3}, \quad \rho=\frac{\lambda_{\mathrm{w}} \Gamma}{4 \pi},
$$

where $\lambda_{\mathrm{w}}$ is the undulator period, $K=e \lambda_{\mathrm{w}} H_{\mathrm{w}} / 2 \pi m c^{2}$ is the undulator parameter, $H_{\mathrm{w}}$ is the undulator magnetic field, $j_{0}$ is the beam current density, $(-e)$ and $m$ are the electron charge and mass, $I_{A}=m c^{3} / e \simeq 17 k A, \omega$ is the radiation frequency, $A_{J J}=J_{0}(\nu)-J_{1}(\nu)$ and $\nu=K^{2} /\left(4+2 K^{2}\right)$. In this paper all formulae are written for the case of a planar undulator. 
In the previous section we have shown that due to the shot noise there is density modulation of the electron beam at the undulator entrance. It follows from the steady-state theory of the FEL amplifier that when the electron beam is modulated at frequency $\omega$ at the undulator entrance, this initiates the amplification process of the electromagnetic wave having the same frequency. One-dimensional model assumes that the plane electromagnetic wave $E=\tilde{E}(z) \exp [i \omega(z / c-t)]+C . C$. is amplified in the FEL amplifier. In the linear high-gain regime the amplitude of the electromagnetic field grows exponentially with the undulator length:

$$
\tilde{E}(z) \propto \exp [\Lambda z]
$$

where $\Lambda$ is the growing root of the eigenvalue equation $(\operatorname{Re} A>0)$ :

$$
A(\Lambda+i C)^{2}=i \Gamma^{3} \text {. }
$$

Here $C=\left[2 \pi / \lambda_{\mathrm{w}}-\omega\left(1+K^{2} / 2\right) /\left(2 c \gamma^{2}\right)\right]$ is the detuning of the particle from resonance. Near the exact resonance, at $|C| \ll \Gamma$, we have the following solution for the growing root (see, e.g., ref. [21]):

$$
\operatorname{Re} \hat{\Lambda}=\frac{\sqrt{3}}{2}\left(1-\frac{\hat{C}^{2}}{9}\right), \quad \operatorname{Im} \hat{\Lambda}=\frac{1}{2}\left(1-\frac{4 \hat{C}}{3}\right),
$$

where $\hat{\Lambda}=\Lambda / \Gamma, \hat{C}=C / \Gamma=\left(\omega_{0}-\omega\right) / 2 \rho \omega_{0}$ is the detuning parameter and $\omega_{0}=$ $4 \pi c \gamma^{2} /\left[\lambda_{\mathbf{w}}\left(1+K^{2} / 2\right)\right]$ is the resonance frequency.

When modulated electron beam is fed to the undulator entrance, the solution of the initial-value problem in a high-gain limit has the form at $\omega>0$ (see, e.g. ref. [21], Appendix $\mathrm{B})$ :

$$
\frac{\bar{E}(\omega, \hat{z})}{E_{0}}=\frac{1}{3} \exp \left(i \frac{\omega}{c} z\right) \exp \left[\frac{\sqrt{3}}{2}\left(1-\frac{\hat{C}^{2}}{9}\right) \hat{z}+\frac{i}{2}\left(1-\frac{4 \hat{C}}{3}\right)\right] \frac{\bar{j}(\omega)}{j_{0}},
$$

where $\bar{E}(\omega, z)$ and $\bar{j}(\omega)$ are, respectively, Fourier components of electric field of the wave $E(t, z)$ and of the beam current density $j(t)$ at the undulator entrance and $\hat{z}=\Gamma z$. At $\omega<0$ the Fourier harmonic is defined by relation $\bar{E}^{*}(\omega, \hat{z})=\bar{E}(-\omega, \hat{z})$. Normalizing factors $E_{0}$ and $j_{0}$ are given by the expressions:

$$
E_{0}=8 \pi \rho^{2} \gamma^{2} I_{A} /\left(\lambda_{\mathrm{w}} c K A_{J J}\right), \quad j_{0}=I_{0} / S,
$$

where $S$ is the transverse area of the electron beam.

In the framework of accepted approximations we can consider Fourier component of the input signal in eq. (2.16) as a Fourier component of the shot noise signal. Transversely coherent fraction of the input shot noise signal is defined by the total beam current [18], so

$$
\frac{\bar{j}(\omega)}{j_{0}}=\frac{\bar{I}(\omega)}{I_{0}}
$$


where $I_{0}$ is the average beam current. For the case of rectangular bunch $I_{0}=(-e) N / T$.

Using eqs. (2.16) and (2.17) we can write:

$$
<|\bar{E}(\omega)|^{2}>=A \exp \left[-\frac{\left(\omega-\omega_{0}\right)^{2}}{2 \sigma_{A}^{2}}\right]<|\bar{I}(\omega)|^{2}>, \quad \omega>0
$$

where

$$
A=\frac{1}{9}\left(\frac{E_{0}}{I_{0}}\right)^{2} \exp (\sqrt{3} \hat{z}), \quad \sigma_{A}=3 \sqrt{\frac{2}{\sqrt{3}}} \frac{\rho \omega_{0}}{\sqrt{\hat{z}}} .
$$

In the following we will analyze in detail the case of rectangular profile of the electron bunch and assume pulse duration $T$ to be large,

$$
\sigma_{A} T \gg 1
$$

It follows from eq. (2.19) that the FEL amplifier bandwidth $\sigma_{A}$ is of the order of $\rho \omega_{0}$, so condition $(2.20)$ can be rewritten as follows

$$
\rho \omega_{0} T \gg 1
$$

This relation means that the steady-state Green's function (2.16) can be used for calculation of the Fourier harmonics of the output field. The value $\rho \omega_{0} T$ is of the order of $10^{2} \div 10^{3}$ for modern projects of VUV and X-ray SASE FELs $[5,6,8,9]$, so the obtained result can be used for practical calculations.

\subsection{Analysis of radiation properties in frequency domain}

Fourier amplitude of the electric field at the exit of SASE FEL operating in the linear high-gain regime can be written as follows:

$$
\bar{E}(\omega)=H_{A}\left(\omega-\omega_{0}\right) \bar{I}(\omega), \quad \omega>0,
$$

where $H_{A}\left(\omega-\omega_{0}\right)$ is the Green's function (see eqs. (2.16) and (2.18)). For many applications and for diagnostic measurements of SASE FEL radiation a monochromator will be installed at the FEL amplifier exit. In the present treatment we assume that such a monochromator has symmetric transmission function $H_{m}\left(\omega-\omega_{0}\right)$ with central frequency $\omega_{0}$ corresponding to the maximum of the FEL gain curve. In this case the expression for $\bar{E}(\omega)$ takes the form:

$$
\bar{E}(\omega)=H_{m}\left(\omega-\omega_{0}\right) H_{A}\left(\omega-\omega_{0}\right) \bar{I}(\omega), \quad \omega>0,
$$

It follows from eq. (2.23) that statistical properties of the Fourier amplitudes $\bar{E}(\omega)$ are defined by statistical properties of the Fourier amplitudes of input current $\bar{I}(\omega)$. In particular, it follows immediately from eq. (2.4) that $|\bar{E}(\omega)|^{2}$ is distributed in accordance with the exponential probability density function: 


$$
p\left(|\tilde{E}(\omega)|^{2}\right)=\frac{1}{\left.<|\bar{E}(\omega)|^{2}\right\rangle} \exp \left(-\frac{|\bar{E}(\omega)|^{2}}{\left.<|\bar{E}(\omega)|^{2}\right\rangle}\right) .
$$

It should be noted that such a distribution is the feature of completely chaotic polarized radiation (see ref. [19] for more detail).

The first order spectral correlations. The first order spectral correlation function is defined as

$$
g_{1}\left(\omega, \omega^{\prime}\right)=\frac{<\bar{E}(\omega) \bar{E}^{*}\left(\omega^{\prime}\right)>}{\left[<|\bar{E}(\omega)|^{2}><\left|\bar{E}\left(\omega^{\prime}\right)\right|^{2}>\right]^{1 / 2}} .
$$

Substituting eqs. (2.23) and (2.8) into eq. (2.25) we obtain analytical expression for the first order spectral correlation function of the FEL amplifier with rectangular electron bunch:

$$
g_{1}\left(\omega, \omega^{\prime}\right)=\bar{F}\left(\omega-\omega^{\prime}\right)=\frac{\sin \frac{\left(\omega-\omega^{\prime}\right) T}{2}}{\frac{\left(\omega-\omega^{\prime}\right) T}{2}} .
$$

We define the spectral coherence as follows:

$$
\Delta \omega_{c}=\int_{-\infty}^{\infty}\left|g_{1}\left(\omega-\omega^{\prime}\right)\right|^{2} d\left(\omega-\omega^{\prime}\right) .
$$

The value of the spectral coherence for the case of rectangular bunch is equal to:

$$
\Delta \omega_{c}=\frac{2 \pi}{T} \text {. }
$$

The second order spectral correlations. The second order spectral correlation function is defined as

$$
g_{2}\left(\omega, \omega^{\prime}\right)=\frac{\left.<|\bar{E}(\omega)|^{2}\left|\bar{E}\left(\omega^{\prime}\right)\right|^{2}\right\rangle}{\left.<|\bar{E}(\omega)|^{2}\right\rangle\left\langle\left|\bar{E}\left(\omega^{\prime}\right)\right|^{2}\right\rangle} .
$$

Using eqs. (2.23), (2.11) and (2.29) we obtain that the first and the second order correlation functions are connected by the relation:

$$
g_{2}\left(\omega, \omega^{\prime}\right)=1+\left|g_{1}\left(\omega, \omega^{\prime}\right)\right|^{2},
$$

which is also general property of completely chaotic polarized radiation. Explicit expression for the second order spectral correlation function of the FEL amplifier with rectangular electron bunch has the form:

$$
g_{2}\left(\omega, \omega^{\prime}\right)=1+\frac{\sin ^{2}\left[\frac{\left(\omega-\omega^{\prime}\right) T}{2}\right]}{\frac{\left(\omega-\omega^{\prime}\right)^{2} T^{2}}{4}} .
$$


Fluctuations of the radiation energy at the exit of the monochromator. The next problem is description of the fluctuations of the radiation bunch energy $W$ at a detector installed after a monochromator with finite bandwidth. From the expression for Pointing's vector and Parseval's theorem we have

$$
W=\frac{c S}{4 \pi^{2}} \int_{0}^{\infty}|\bar{E}(\omega)|^{2} d \omega
$$

Taking into account eq. (2.23) we can write the expression for the average energy measured by the detector:

$$
\left.<W>=\frac{c S}{4 \pi^{2}} \int_{0}^{\infty}<|\bar{E}(\omega)|^{2}\right\rangle d \omega=\frac{c e^{2} S N}{4 \pi^{2}} \int_{0}^{\infty}\left|H_{m}\left(\omega-\omega_{0}\right)\right|^{2}\left|H_{A}\left(\omega-\omega_{0}\right)\right|^{2} d \omega .
$$

It is seen that the average energy is the function of the frequency profile of the monochromator and of the gain profile of the FEL amplifier.

The normalized dispersion of the energy distribution is calculated as follows:

$$
\sigma_{W}^{2}=\frac{\left\langle\left(W-\langle W>)^{2}\right\rangle\right.}{\langle W\rangle^{2}}=\frac{\left.\int_{0}^{\infty} d \omega \int_{0}^{\infty} d \omega^{\prime}<|\bar{E}(\omega)|^{2}\left|\bar{E}\left(\omega^{\prime}\right)\right|^{2}\right\rangle}{\left.\int_{0}^{\infty} d \omega<|\bar{E}(\omega)|^{2}\right\rangle \int_{0}^{\infty} d \omega^{\prime}\left\langle\left|\bar{E}\left(\omega^{\prime}\right)\right|^{2}\right\rangle}-1 .
$$

Using definition (2.29) of the second order correlation function and relation (2.30) we reduce this expression to the form:

$$
\sigma_{W}^{2}=\frac{\int_{0}^{\infty} d \omega \int_{0}^{\infty} d \omega^{\prime}<|\bar{E}(\omega)|^{2}><\left|\bar{E}\left(\omega^{\prime}\right)\right|^{2}>\left|g_{1}\left(\omega^{\prime}, \omega^{\prime}\right)\right|^{2}}{\int_{0}^{\infty} d \omega<|\bar{E}(\omega)|^{2}>\int_{0}^{\infty} d \omega^{\prime}<\left|\bar{E}\left(\omega^{\prime}\right)\right|^{2}>} .
$$

Analysis of this expression shows that the energy deviation after monochromator is function of the frequency profile of the monochromator, of the gain profile of the FEL amplifier and of the electron bunch form factor.

Let us derive analytical expressions for the energy dispersion after the monochromator for the cases of a Gaussian and rectangular profile of the monochromator line. Rectangular line of monochromator is defined as $(\omega>0)$ :

$$
\begin{array}{lll}
\left|H_{m}\left(\omega-\omega_{0}\right)\right|^{2}=1 & \text { at } & \left|\omega-\omega_{0}\right|<\frac{\Delta \omega_{\mathrm{m}}}{2} \\
\left|H_{m}\left(\omega-\omega_{0}\right)\right|^{2}=0 & \text { at } & \left|\omega-\omega_{0}\right|>\frac{\Delta \omega_{\mathrm{m}}}{2},
\end{array}
$$

and the Gaussian line of the monochromator is defined as

$$
\left|H_{m}\left(\omega-\omega_{0}\right)\right|^{2}=\frac{1}{\sqrt{2 \pi} \sigma_{m}} \exp \left[-\frac{\left(\omega-\omega_{0}\right)^{2}}{2 \sigma_{m}^{2}}\right] \text {. }
$$


Substituting expressions (2.23), (2.18), (2.26) and (2.36) into eq. (2.35) we obtain the expression for the radiation energy dispersion after monochromator for the case of the rectangular monochromator line:

$$
\begin{aligned}
& \sigma_{W}^{2}=\left[\frac{\pi}{2} \hat{\sigma}_{A}^{2} \operatorname{erf}^{2}\left(\frac{\Delta \hat{\omega}_{m}}{2 \sqrt{2} \hat{\sigma}_{A}}\right)\right]^{-1}\left\{\sqrt{\pi} \hat{\sigma}_{A} \operatorname{erf}\left(\frac{\Delta \hat{\omega}_{m}}{2 \hat{\sigma}_{A}}\right)\left[\operatorname{Si}\left(\Delta \hat{\omega}_{m}\right)-\frac{1-\cos \left(\Delta \hat{\omega}_{m}\right)}{\Delta \hat{\omega}_{m}}\right]\right. \\
& \left.+\exp \left(-\frac{\Delta \hat{\omega}_{m}^{2}}{4 \hat{\sigma}_{A}^{2}}\right)\left[\operatorname{Ci}\left(\Delta \hat{\omega}_{m}\right)-\ln \left(\Delta \hat{\omega}_{m}\right)-G\right]\right\},
\end{aligned}
$$

where $\Delta \hat{\omega}_{m}=\Delta \omega_{m} T, \hat{\sigma}_{A}=\sigma_{A} T$ is $G=0.577 \ldots$ is Euler's constant, $\operatorname{erf}(\ldots)$ is error function, and $\mathrm{Ci}(\ldots)$ and $\mathrm{Si}(\ldots)$ are integral sine and cosine, respectively [22].

The corresponding expression for the case of the Gaussian monochromator line is as follows:

$$
\sigma_{W}^{2}=\frac{\sqrt{\pi}}{\hat{\sigma}^{2}} \int_{0}^{\hat{\sigma}} \operatorname{erf}(x) d x,
$$

where

$$
\hat{\sigma}=\frac{\hat{\sigma}_{A} \hat{\sigma}_{m}}{\sqrt{\hat{\sigma}_{A}^{2}+\hat{\sigma}_{m}^{2}}}, \quad \hat{\sigma}_{m}=\sigma_{m} T .
$$

Let us study asymptotical behavior of the expressions (2.38) and (2.39). When the monochromator line is much narrower than the interval of spectral coherence (2.28), the normalized dispersion tends to unity:

$$
\sigma_{W}^{2} \simeq 1 \quad \text { at }\left(\Delta \omega_{m} T, \sigma_{m} T\right) \ll 1 .
$$

When the monochromator line width is much larger than the spectral interval of coherence and much less than the FEL amplifier bandwidth, the dispersion is inversly proportional to the monochromator line width:

$$
\begin{array}{lll}
\sigma_{W}^{2} \simeq \frac{2 \pi}{\Delta \omega_{m} T} & \text { at } 1 \ll \Delta \omega_{m} T \ll \sigma_{A} T & \text { for rectangular line, } \\
\sigma_{W}^{2} \simeq \frac{\sqrt{\pi}}{\sigma_{m} T} & \text { at } 1 \ll \sigma_{m} T \ll \sigma_{A} T & \text { for Gaussian line } .
\end{array}
$$

When the monochromator line width is much larger than the FEL amplifier bandwidth, the fluctuations are defined by the FEL line width:

$$
\sigma_{W}^{2} \simeq \frac{\sqrt{\pi}}{\sigma_{A} T} \quad \text { at }\left(\Delta \omega_{\mathrm{m}}, \sigma_{\mathrm{m}}\right) \gg \sigma_{\mathrm{A}}
$$

The next practical problem is to find the probability density distribution of the radiation energy after monochromator, $p(W)$. In general, this problem has no exact solution and we use an assumption for an approximate form for such a distribution which will be 
confirmed below with the results of numerical simulations. This assumption is based on the above proved statement that the radiation from SASE FEL possesses all the features of completely chaotic polarized radiation. Using arguments similar to that of ref. [19] (Chapter 6) we suppose that the distribution of the radiation energy after the monochromator is described rather well by a gamma probability density function:

$$
p(W)=\frac{M^{M}}{\Gamma(M)}\left(\frac{W}{<W>}\right)^{M-1} \frac{1}{<W>} \exp \left(-M \frac{W}{<W>}\right),
$$

where $\Gamma(M)$ is gamma function of argument $M$ and

$$
M=\frac{1}{\sigma_{W}^{2}} .
$$

This distribution provides correct values for the mean value of $W$ and for the dispersion $\sigma_{W}^{2}=1 / M$ :

$$
\int_{0}^{\infty} W p(W) d W=<W>, \quad \int_{0}^{\infty} \frac{(W-<W>)^{2}}{<W>^{2}} p(W) d W=\frac{1}{M} .
$$

Parameter $M$ can be interpreted as the average number of "degrees of freedom" or "modes" in a radiation pulse. It follows from eq. (2.35) that this parameter can not be less than unity. When $M$ tends to unity, the distribution (2.43) tends to the negative exponential distribution (2.24). When $M \gg 1$, the distribution (2.43) tends to the Gaussian distribution.

\subsection{Analysis of the radiation properties in time domain}

In the previous section we described statistical properties of the output SASE FEL radiation in the frequency domain. In this section we perform similar investigation in the time domain.

Let us start with calculation of slowly varying complex amplitude $\tilde{E}(t)$ of the electric field at a given distance from the undulator entrance, $\hat{z}=\Gamma z$. Using expressions (2.22) and (2.16) we obtain:

$$
\frac{\tilde{E}(t)}{E_{0}}=\frac{\rho \omega_{0} T}{\sqrt{\sqrt{3} \pi \hat{z}}} \exp \left[(\sqrt{3}+i) \frac{\hat{z}}{2}\right] \frac{1}{N} \sum_{k=1}^{N} \exp \left(i \omega_{0} t_{k}\right) \exp \left[-\frac{\sigma_{A}^{2}\left(t-t_{z}-t_{k}\right)^{2}}{2}\right],
$$

where

$$
t_{z}=\frac{z}{c}\left(1+\frac{1+K^{2} / 2}{3 \gamma^{2}}\right),
$$

and $\sigma_{A}$ is defined by expression (2.19). Since $\sigma_{A} \simeq \rho \omega_{0}$ is much less than $\omega_{0}$, we can approximately let the amplitude and the phase of each random phasor contributing to the sum in eq. (2.45) to be independent of each other, and phases to be uniformly distributed 
on interval $(-\pi, \pi)$. Thus, with such an accuracy the distribution of the instantaneous radiation power $P \propto|\tilde{E}|^{2}$ is the negative exponential distribution:

$$
p\left(|\tilde{E}(t)|^{2}\right)=\frac{1}{\left\langle|\tilde{E}(t)|^{2}\right\rangle} \exp \left(-\frac{|\tilde{E}(t)|^{2}}{\left\langle|\tilde{E}(t)|^{2}\right\rangle}\right) .
$$

The first order time correlations. The correlation between the radiation fields at time moments $t$ and $t^{\prime}$ has the form:

$$
\begin{aligned}
& <\tilde{E}(t) \tilde{E}^{*}\left(t^{\prime}\right)>\exp \left[-i \omega_{0}\left(t-t^{\prime}\right)\right]= \\
& \frac{1}{4 \pi^{2}} \int_{0}^{\infty} d \omega \int_{0}^{\infty} d \omega^{\prime} \exp \left(-i \omega t+i \omega^{\prime} t^{\prime}\right)<\bar{E}(\omega) \bar{E}^{*}\left(\omega^{\prime}\right)>= \\
& \left.\frac{1}{4 \pi^{2}} \int_{0}^{\infty} d \omega \int_{0}^{\infty} d \omega^{\prime} \exp \left(-i \omega t+i \omega^{\prime} t^{\prime}\right)\left[<|\bar{E}(\omega)|^{2}\right\rangle\left\langle\left|\bar{E}\left(\omega^{\prime}\right)\right|^{2}\right\rangle\right]^{1 / 2} \bar{F}\left(\omega-\omega^{\prime}\right) .
\end{aligned}
$$

Here formulae (2.25) and (2.26) have been used when rewriting the integral. In this paper we use approximation (2.21) which assumes the interval of spectral coherence, $\Delta \omega_{c}=2 \pi / T$, to be much less than the FEL amplifier bandwidth, $\sigma_{A} \simeq \rho \omega_{0}$. Using this approximation we can simplify the integral in eq (2.47) in the following way. We substitute the expression in square brackets by $<|\bar{E}(\omega)|^{2}>$ and after integration over $\omega$ and $\Delta \omega=\left(\omega-\omega^{\prime}\right)$ we obtain that

$$
\begin{aligned}
& <\tilde{E}(t) \tilde{E}^{*}\left(t^{\prime}\right)>\exp \left[-i \omega_{0}\left(t-t^{\prime}\right)\right] \simeq \\
& \left.\frac{1}{4 \pi^{2}} \int_{-\infty}^{\infty} d(\Delta \omega) \exp (-i \Delta \omega t) \bar{F}(\Delta \omega) \int_{0}^{\infty} d \omega \exp \left[-i \omega\left(t-t^{\prime}\right)\right]<|\bar{E}(\omega)|^{2}\right\rangle= \\
& \left.\frac{F(t)}{2 \pi} \int_{0}^{\infty} d \omega \exp \left[-i \omega\left(t-t^{\prime}\right)\right]<|\bar{E}(\omega)|^{2}\right\rangle,
\end{aligned}
$$

where $F(t)$ is the radiation pulse profile. For slowly varying complex amplitudes the time correlation function of the first order is defined as follows:

$$
g_{1}\left(t-t^{\prime}\right)=\frac{<\tilde{E}(t) \tilde{E}^{*}\left(t^{\prime}\right)>}{\left[<|\tilde{E}(t)|^{2}><\left|\tilde{E}\left(t^{\prime}\right)\right|^{2}>\right]^{1 / 2}} .
$$

Using formula (2.48) we can write

$$
g_{1}\left(t-t^{\prime}\right)=\frac{\int_{0}^{\infty} d \omega<|\bar{E}(\omega)|^{2}>\exp \left[-i\left(\omega-\omega_{0}\right)\left(t-t^{\prime}\right)\right]}{\int_{0}^{\infty} d \omega<|\bar{E}(\omega)|^{2}>} .
$$

Remembering relation (2.22), we rewrite this expression in the following way: 


$$
g_{1}\left(t-t^{\prime}\right)=\frac{\int_{-\infty}^{\infty} d(\Delta \omega)\left|H_{A}(\Delta \omega)\right|^{2} \exp \left[-i \Delta \omega\left(t-t^{\prime}\right)\right]}{\int_{-\infty}^{\infty} d(\Delta \omega)\left|H_{A}(\Delta \omega)\right|^{2}}
$$

where $\Delta \omega=\left(\omega-\omega_{0}\right)$. It follows from this expression that the correlation function of the first order, $g_{1}$, has the real part even with respect to $\left(t-t^{\prime}\right)$ and the imaginary part odd with respect to $\left.\left(t-t^{\prime}\right)\right)$. When the FEL gain curve is symmetric with respect to the resonance frequency, $\omega_{0}$, function $g_{1}$ is real. In the high-gain linear regime the FEL gain function $\left|H_{A}(\Delta \omega)\right|^{2}$ is symmetric (see eq. (2.18)), and we obtain the following expression for the first order time correlation function:

$$
g_{1}(\tau)=\exp \left(-\frac{9 \rho^{2} \omega_{0}^{2} \tau^{2}}{\sqrt{3} \hat{z}}\right)
$$

where $\tau=\left(t-t^{\prime}\right)$. Following the approach of ref. [24], we define the coherence time $\tau_{c}$ as

$$
\tau_{c}=\int_{-\infty}^{\infty}\left|g_{1}(\tau)\right|^{2} d \tau
$$

When SASE FEL operates in the high gain linear mode the explicit expression for the coherence time is

$$
\tau_{c}=\frac{\sqrt{\pi}}{\sigma_{A}}=\sqrt{\frac{\sqrt{3} \pi \hat{z}}{18}} \frac{1}{\rho \omega_{0}} .
$$

As a rule, the Michelson interferometer is used for the measurement of the first order time correlation function, $g_{1}(\tau)$. Changing the pathlength difference in the scheme of interferometer one gets the interferogram - the dependence of the average (shot to shot) intensity on the pathlength difference. The module and the phase of the first order correlation function are reconstructed using this interferogram.

It should be noted that the FEL gain curve can be reconstructed from the first order time correlation function. Indeed, functions $g_{1}(\tau)$ and $\left|H_{A}(\Delta \omega)\right|^{2}$ are connected by the Fourier transformation

$$
\frac{\left|H_{A}(\Delta \omega)\right|^{2}}{\int_{-\infty}^{\infty} d(\Delta \omega)\left|H_{A}(\Delta \omega)\right|^{2}}=\frac{1}{2 \pi} \int_{-\infty}^{\infty} d \tau g_{1}(\tau) \exp (-i \Delta \omega \tau)
$$

Such a technique for spectrum reconstruction is named as Fourier spectroscopy and is widely used for optical measurements.

The second order time correlations. The correlation between the radiation intensities at time moments $t$ and $t^{\prime}$ has the form: 


$$
\begin{aligned}
& <|\tilde{E}(t)|^{2}\left|\tilde{E}\left(t^{\prime}\right)\right|^{2}>=\frac{1}{16 \pi^{4}} \int_{0}^{\infty} d \omega_{1} \int_{0}^{\infty} d \omega_{2} \int_{0}^{\infty} d \omega_{3} \times \\
& \int_{0}^{\infty} d \omega_{4} \exp \left[-i\left(\omega_{1}-\omega_{2}\right) t+i\left(\omega_{3}-\omega_{4}\right) t^{\prime}\right]<\bar{E}\left(\omega_{1}\right) \vec{E}\left(\omega_{3}\right) \bar{E}^{*}\left(\omega_{2}\right) \bar{E}^{*}\left(\omega_{4}\right)>.
\end{aligned}
$$

Taking into account expressions (2.22) and (2.7) we can simplify the correlation in the integrand of eq. (2.55) in the following way:

$$
\begin{aligned}
& <\bar{E}\left(\omega_{1}\right) \bar{E}\left(\omega_{3}\right) \bar{E}^{*}\left(\omega_{2}\right) \bar{E}^{*}\left(\omega_{4}\right)>=<\bar{E}\left(\omega_{1}\right) \bar{E}^{*}\left(\omega_{2}\right)><\bar{E}\left(\omega_{3}\right) \bar{E}^{*}\left(\omega_{4}\right)>+ \\
& +<\bar{E}\left(\omega_{1}\right) \bar{E}^{*}\left(\omega_{4}\right)><\bar{E}\left(\omega_{3}\right) \bar{E}^{*}\left(\omega_{2}\right)>
\end{aligned}
$$

and present integral (2.55) as a sum of two terms. To calculate the first term one should take into account eqs. $(2.25),(2.26),(2.48)$ and $(2.21)$ which leads to

$$
\begin{aligned}
& \frac{1}{16 \pi^{4}} \int_{0}^{\infty} d \omega_{1} \int_{0}^{\infty} d \omega_{2} \exp \left[-i\left(\omega_{1}-\omega_{2}\right) t\right]<\bar{E}\left(\omega_{1}\right) \bar{E}^{*}\left(\omega_{2}\right)>\times \\
& \frac{1}{16 \pi^{4}} \int_{0}^{\infty} d \omega_{3} \int_{0}^{\infty} d \omega_{4} \exp \left[-i\left(\omega_{3}-\omega_{4}\right) t^{\prime}\right]<\bar{E}\left(\omega_{3}\right) \bar{E}^{*}\left(\omega_{4}\right)>= \\
& \left.\frac{1}{16 \pi^{4}} \int_{0}^{\infty} d \omega_{1} \int_{0}^{\infty} d \omega_{2} \exp \left[-i\left(\omega_{1}-\omega_{2}\right) t\right]\left[<\left|\bar{E}\left(\omega_{1}\right)\right|^{2}><\left|\bar{E}\left(\omega_{2}\right)\right|^{2}\right\rangle\right]^{1 / 2} \bar{F}\left(\omega_{1}-\omega_{2}\right) \times \\
& \int_{0}^{\infty} d \omega_{3} \int_{0}^{\infty} d \omega_{4} \exp \left[-i\left(\omega_{3}-\omega_{4}\right) t^{\prime}\right]\left[\left\langle\left|\bar{E}\left(\omega_{3}\right)\right|^{2}\right\rangle\left\langle\left|\bar{E}\left(\omega_{4}\right)\right|^{2}\right\rangle\right]^{1 / 2} \bar{F}\left(\omega_{3}-\omega_{4}\right) \simeq \\
& \frac{1}{16 \pi^{4}} \int_{0}^{\infty} d \omega<|\bar{E}(\omega)|^{2}>\int_{0}^{\infty} d(\Delta \omega) \exp (-i \Delta \omega t) \bar{F}(\Delta \omega) \times \\
& \int_{0}^{\infty} d \omega^{\prime}<\left|\bar{E}\left(\omega^{\prime}\right)\right|^{2}>\int_{0}^{\infty} d\left(\Delta \omega^{\prime}\right) \exp \left(-i \Delta \omega^{\prime} t^{\prime}\right) \bar{F}\left(\Delta \omega^{\prime}\right)= \\
& <|\tilde{E}(t)|^{2}><\left|\tilde{E}\left(t^{\prime}\right)\right|^{2}>.
\end{aligned}
$$

In the same way we obtain the expression for the second term. Finally we obtain:

$$
<|\tilde{E}(t)|^{2}\left|\tilde{E}\left(t^{\prime}\right)\right|^{2}>=<|\tilde{E}(t)|^{2}><\left|\tilde{E}\left(t^{\prime}\right)\right|^{2}>+\left|<\tilde{E}(t) \tilde{E}^{*}\left(t^{\prime}\right)>\right|^{2}
$$

The second order time correlation function is defined as follows:

$$
g_{2}\left(t-t^{\prime}\right)=\frac{\left.<|\tilde{E}(t)|^{2}\left|\tilde{E}\left(t^{\prime}\right)\right|^{2}\right\rangle}{\left.<|\tilde{E}(t)|^{2}><\left|\tilde{E}\left(t^{\prime}\right)\right|^{2}\right\rangle} .
$$

It follows from eq. (2.58) and definitions of $g_{1}\left(t-t^{\prime}\right)$ and $g_{2}\left(t-t^{\prime}\right)$ that

$$
g_{2}\left(t-t^{\prime}\right)=1+\left|\cdot g_{1}\left(t-t^{\prime}\right)\right|^{2}
$$


For the SASE FEL operating in the high-gain linear regime the explicit expression for the second order time correlation function is (see eqs. (2.51) and (2.60)):

$$
g_{2}(\tau)=1+\exp \left(-\frac{18 \rho^{2} \omega_{0}^{2} \tau^{2}}{\sqrt{3} \hat{z}}\right)
$$

Relations (2.46) and (2.60) shows that the SASE FEL radiation is completely chaotic polarized radiation (see also relations (2.24) and (2.30) obtained in frequency domain).

Fluctuations of the energy in the radiation pulse. The next problem is the description of the fluctuations of the radiation energy $W$ detected during finite time interval $\delta T$ :

$$
W=\int_{t}^{t+\delta T} P(t) d t .
$$

It can be shown that such a distribution is described rather well by gamma probability density function (2.43) with parameter $M$ equal to [19]:

$$
M^{-1}=\sigma_{W}^{2}=\frac{\sqrt{\pi}}{\delta \hat{T}} \operatorname{erf}(\delta \hat{\mathrm{T}})-\frac{1}{(\delta \hat{\mathrm{T}})^{2}}\left[1-\mathrm{e}^{-(\delta \hat{\mathrm{T}})^{2}}\right]
$$

where $\delta \hat{T}=\delta T \sigma_{A}$. When $\delta T$ is less than coherence time $\tau_{c}(2.53)$, parameter $M$ tends to unity and gamma distribution tends to negative exponential distribution. In opposite case, when $\delta T \gg \tau_{c}$ we can write:

$$
M^{-1}=\frac{\sqrt{\pi}}{\delta T \sigma_{A}}=\frac{\tau_{c}}{\delta T},
$$

and gamma distribution tends to a Gaussian one. At $\delta T=T$ we obtain relation (2.42).

Let us discuss the problem how to measure statistical properties of the SASE FEL radiation. Typical pulse duration of existent projects of SASE FELs is of about a fraction of picosecond. The resolution time of modern fast photoelectric detectors is much larger than this value, of about a fraction of nanosecond, which allows to measure total energy of the radiation pulse only. So, the measurement of the finite-time integrated instantaneous power seems to be technically impossible. On the other hand, there is no such evident technical limitations for measurement of statistical properties of SASE FEL radiation in frequency domain. At sufficient resolution of the monochromator it seems to be technically feasible to measure the first and the second order spectral correlation function as well as the distribution of the radiation energy fluctuations at the exit of the monochromator.

\section{Photoelectric Detection of SASE FEL Radiation}

In this section we study the problem of photocount fluctuations in the detector of the SASE FEL radiation. We assume that the SASE FEL radiation has full transverse coherence and time characteristics of radiation follow the laws described in the previous 
section. It has been shown above that the energy, $W$, in the radiation pulse reaching the photodetector is unpredictable, we can predict the probability density $p(W)$ only. In this case the probability of detection of $K$ photons is given by Mandel's semiclassical formula [19]:

$$
P(K)=\int_{0}^{\infty} \frac{(\alpha W)^{K}}{K !} \exp (-\alpha W) p(W) d W,
$$

where $\alpha=\eta / \hbar \omega_{0}$ and $\eta$ is the quantum efficiency of the photodetector. Using formula (3.1) we get the expressions for the mean and for the variance of $K$ value [19]:

$$
<K>=\alpha<W>, \quad \sigma_{K}^{2}=\frac{\left.<K^{2}>-<K\right\rangle^{2}}{<K>^{2}}=\frac{1}{<K>}+\sigma_{W}^{2},
$$

where $\sigma_{W}^{2}=1 / M$ is given by formula (2.34). The expression for photocounts fluctuations contains two terms. The first term corresponds to "photon shot noise". The second one corresponds to the classical fluctuations of the energy in the radiation bunch and takes its origin from shot noise in electron bunch. The ratio of the classical variance to the "photon shot noise" variance is equal to

$$
\delta_{c}=\frac{<K>}{M} \text {. }
$$

Parameter $\delta_{c}$ is named as the photocount degeneracy parameter.

Let us consider a scheme with the monochromator installed in front of the photodetector. When monochromator linewidth is large, $\Delta \omega_{m} \gg \Delta \omega_{c}$, parameter $M$ is equal to the number of coherence intervals inside the monochromator linewidth and parameter $\delta_{c}$ can be interpreted as average number of photons detected within the coherence interval $\Delta \omega_{c}$ :

$$
\delta_{c} \simeq \frac{\Delta \omega_{c}}{\Delta \omega_{m}}<K>\quad \text { at } \quad \Delta \omega_{\mathrm{m}} \gg \Delta \omega_{\mathrm{c}} .
$$

In opposite case, at $\Delta \omega_{m} \ll \Delta \omega_{c}$, the value of parameter $M$ is close to unity and parameter $\delta_{c}$ is equal to the average number of detected photons:

$$
\delta_{\mathrm{c}} \simeq\langle K\rangle \quad \text { at } \quad \Delta \omega_{\mathrm{m}} \ll \Delta \omega_{\mathrm{c}} .
$$

When SASE FEL operates in the linear regime, the probability density of the energy after monochromator, $p(W)$, is the gamma distribution (2.43). Substituting (2.43) into Mandel's formula (3.1) and performing integration we come to the negative binomial distribution [19]:

$$
P(K)=\frac{\Gamma(K+M)}{\Gamma(K+1) \Gamma(M)}\left(1+\frac{M}{<K>}\right)^{-K}\left(1+\frac{<K>}{M}\right)^{-M} .
$$

When parameter $M$ tends to unity, the negative binomial distribution transforms to Bose distribution: 


$$
\lim _{M \rightarrow 1} P(K)=\frac{<K>^{K}}{(<K>+1)^{K+1}} .
$$

It means that the number of photons detected after the monochromator with the linewidth $\Delta \omega_{m} \ll \Delta \omega_{c}$ is described approximately with Bose distribution (3.5).

It can be shown that the negative binomial distribution tends to the gamma distribution (2.43) at large values of the count degeneracy parameter $\delta_{c}$. In particular, Bose distribution tends to the negative exponential distribution $\left(\delta_{c} \simeq<K>\right.$ in this case):

$$
\lim _{<K>\rightarrow \infty} \frac{<K>^{K}}{(<K>+1)^{K+1}}=\frac{1}{<K>} \exp \left(-\frac{K}{<K>}\right) .
$$

For the further consideration we introduce the notion of the wave degeneracy parameter $\delta_{W}$ which is equal to the average number of photons radiated by the SASE FEL inside the spectral interval of coherence $\Delta \omega_{c}$. Physically this parameter means the average number of photons which can interfere, or, according to the quantum theory, the number of photons in one quantum state (one "mode"). The wave degeneracy parameter can be calculated as follows

$$
\delta_{W}=\frac{B \lambda^{3}}{4 c}
$$

where $B$ is the peak spectral brightness (or brilliance) of the radiation source and $\lambda$ is the wavelength. The following definition of spectral brightness is used for transversely coherent radiation:

$$
B=\frac{4 \Delta \dot{N}_{p h}}{\lambda \Delta \lambda}
$$

where $\Delta \dot{N}_{p h}$ is the photon flux within the spectral interval $\Delta \lambda / \lambda$. When writing down expression (3.7) the following definition for the emittance of the diffractionally limited photon beam has been used (see, e.g. ref. [23]):

$$
\min \left(\varepsilon_{x, z}\right)=\min \left(2 \pi \sigma_{x, z} \sigma_{x^{\prime}, z^{\prime}}\right)=\frac{\lambda}{2} .
$$

Substituting expression (3.7) into eq. (3.6) we obtain:

$$
\delta_{W}=\frac{\lambda^{2}}{c} \frac{\Delta \dot{N}_{p h}}{\Delta \lambda} .
$$

The spectrum bandwidth $\Delta \lambda / \lambda$ is connected with the coherence time as ${ }^{1}$

$$
\tau_{c}=\frac{\lambda^{2}}{c \Delta \lambda}
$$

Thus we see that parameter $\delta_{W}$ is equal to average number of photons radiated during coherence time $\tau_{c}[19]$.

\footnotetext{
${ }^{1}$ There exists different definition when the spectrum bandwidth is defined as full width at half maximum of the spectrum (FWHM). These definitions agree well with each other. For instance, for the Gaussian shape of the spectrum eq. (3.8) and FWHM definitions differ by a factor of $(2 \ln 2 / \pi)^{1 / 2} \simeq 0.7$ only.
} 
Peculiar feature of SASE FEL is that the wave degeneracy parameter is always extremely large. Let us consider specific example of VUV SASE FEL at DESY [9]. At the wavelength of $\lambda=70 \mathrm{~nm}$ the spectral brightness (in practical units) is of about

$$
B \simeq 10^{30} \text { phot. } /\left(\sec \times \operatorname{mrad}^{2} \times \mathrm{mm}^{2} \times 0.1 \% \text { bandw. }\right)
$$

Substituting this value into formula (3.6) we obtain that parameter $\delta_{W} \simeq 10^{14}$. Typical values of $\delta_{W}$ will be about $10^{10} \div 10^{12}$ for X-ray SASE FELs.

The formula for calculation of the photocount degeneracy parameter $\delta_{c}$ is given by

$$
\delta_{c}=\eta T_{m} \frac{\Delta \omega_{m}}{M \Delta \omega_{c}} \delta_{W},
$$

where $T_{m}$ is the transmission factor of the monochromator. Assuming the monochromator linewidth to be of about $\Delta \omega_{m} / \omega \simeq 10^{-4}$ and rms bunch length to be $\sigma_{z} \simeq 200 \mu \mathrm{m}$, we obtain that the monochromator linewidth corresponds to one interval of coherence, i.e.

$$
\Delta \omega_{m} \simeq \Delta \omega_{c}, \quad M \simeq 1 .
$$

Assuming that $\eta \simeq T_{m} \simeq 10^{-1}$ we estimate the value of count degeneracy parameter to be $\delta_{c} \simeq 10^{12}$ for the $70 \mathrm{~nm}$ SASE FEL at DESY. In accordance with expression (3.3) the classical fluctuations are $10^{12}$ times larger than "photon shot noise" fluctuations.

On the base of consideration performed in this section we can state that classical approach is adequate for description of statistical properties of the radiation from SASE FEL, and experimental investigations of these properties can be performed and interpreted in the frame of classical theory.

\section{Algorithms for numerical simulation of SASE FEL operation}

Analytical considerations presented above refer only to the high gain linear mode of the SASE FEL operation. To obtain more detailed information about the properties of the SASE FEL operating in linear and nonlinear regime, numerical simulation codes should be developed. Analytical results serves as primary standard for testing numerical simulation codes.

\subsection{Method for time-dependent simulation}

Time-dependent algorithm for the simulation of the FEL amplifier should take into account the slippage effect which connected with the fact that electromagnetic wave moves with the velocity of light $c$, while the electron beam moves with the longitudinal velocity $v_{\mathbf{z}}$. Electron motion in the undulator is a periodic one, so the radiation of each electron $E(z, t)$ is also periodic function: 


$$
E(z, t)=f(z-c t)=f(z-c t+\lambda)
$$

with period

$$
\lambda=\lambda_{\mathrm{w}} \frac{c-v_{\mathrm{z}}}{v_{\mathrm{z}}} \simeq \frac{\lambda_{\mathrm{w}}}{2 \gamma_{\mathrm{z}}^{2}}=\lambda_{\mathrm{w}} \frac{1+K^{2} / 2}{2 \gamma^{2}} .
$$

It seems to be natural to construct the following algorithm [16]. Suppose, we have electron bunch of length $l_{\mathrm{b}}$. We divide this length into $N_{\mathrm{b}}=l_{\mathrm{b}} / \lambda$ boxes. FEL equations are used in each box for calculation of the motion of the electrons and evolution of the radiation field within one undulator period, i.e. within $\Delta \hat{z}=\lambda_{\mathrm{w}} \Gamma$. The using of steadystate FEL equations averaged over undulator period is justified by the fact that FEL amplifier is resonance device with a narrow bandwidth (see eq. (2.18) remembering that $\rho \ll 1$ ). Then we should take into account the slippage effect, i.e. that electromagnetic radiation advances the electron beam by the wavelength $\lambda$ while electron beam passes one undulator period. It means that the radiation which interacted with the electrons in the $j$ th box slips to the electrons located in the next, $j+1$ th box. Then procedure of integration is repeated, etc.

This algorithm allows us to calculate the values of radiation field for each box as function of longitudinal coordinate $z$. Time dependence of the radiation field has the form:

$$
E(z, t)=\tilde{E}(z, t) e^{-i \omega_{0}(z / c-t)}+C . C .
$$

at any position along the undulator. Here we explicitly segregated slowly varying complex amplitude $\tilde{E}(z, t)$. At any fixed point $z$ along the undulator the time interval between the arrival of the radiation connected with adjacent boxes is equal to $\Delta t=t_{j+1}-t_{j}=\lambda / \mathrm{c}$, so we have discrete representation of $\tilde{E}\left(z, t_{j}\right)$.

To calculate spectral characteristics we use Fourier transformation:

$$
\begin{aligned}
& E(z, t)=\frac{1}{2 \pi} \int_{-\infty}^{\infty} \bar{E}(z, \omega) e^{-i \omega t} d \omega \\
& \bar{E}(z, \omega)=\int_{-\infty}^{\infty} e^{i \omega t}\left[\tilde{E}(z, t) e^{-i \omega_{0} t}+C . C .\right] d t .
\end{aligned}
$$

Taking into account that the radiation field $E(z, t)$ is calculated in discrete time moments, we can write:

$$
\bar{E}(z, \omega)=\frac{2 \pi}{\omega_{0}} \sum_{j} \tilde{E}^{(j)} e^{-2 \pi i j \Delta \omega / \omega_{0}}
$$

where $\Delta \omega=\omega-\omega_{0}$ and $\omega>0$.

The spectral density of the radiation energy is given by the expression: 


$$
\frac{d W}{d(\Delta \omega)}=\frac{c S}{4 \pi^{2}} \bar{E}(z, \Delta \omega) \bar{E}^{*}(z, \Delta \omega)
$$

which is related with the total radiation energy in the pulse as:

$$
W=\frac{c S}{4 \pi} \int_{0}^{T} E^{2}(z, t) d t=\int_{-\infty}^{\infty} \frac{d W}{d(\Delta \omega)} d(\Delta \omega),
$$

The first and the second order time correlation functions, $g_{1}(\tau)$ and $g_{2}(\tau)$, are calculated in accordance with the definitions given by relations (2.49) and (2.59).

\subsection{Linear simulation code}

All formulae, presented below, are written for the case of stepped axial profile of electron beam. Extending of these formulae to the case of an arbitrary gradient axial profile could be performed in a simple manner.

When performing numerical simulations, we neglect the effects of the space charge fields and the longitudinal velocity spread in the beam. The length of the electron beam $l_{\mathrm{b}}$ is assumed to be rather large, $l_{\mathrm{b}} \gg\left(1+K^{2} / 2\right) /\left(2 \gamma^{2} \Gamma\right)$. We also assume that the fluctuations of the beam current density define the value of the input shot noise signal. It means that we neglect the effect of the longitudinal velocity fluctuations connected with finite energy spread in the beam. One can show that the ratio of the noise signal due to the fluctuations of the velocity to the shot noise signal is of the order of $\sigma_{E}^{2} / \mathcal{E}^{2} \rho^{2}$, where $\sigma_{E}$ is the energy spread in the beam. This ratio is always less than unity, because the safety margin for the FEL amplifier operation assumes that $\sigma_{E}^{2} / \mathcal{E}^{2} \ll \rho^{2}$.

Linear time-dependent simulation algorithm is organized as follows. We divide the electron beam into $N_{\mathrm{b}}=l_{\mathrm{b}} / \lambda$ boxes. Linear steady-state FEL equations are used in each box to calculate the evolution of the radiation field within one undulator period, i.e. within $\Delta \hat{z}=\lambda_{\mathrm{w}} \Gamma$ (see, e.g. [21]):

$$
\frac{d^{3} \hat{E}^{(j)}}{d \hat{z}^{3}}=i \hat{E}^{(j)}
$$

where $\hat{E}^{(j)}=\tilde{E} / E_{0}$ is the reduced complex amplitude of electric field of the electromagnetic wave in the $j$ th box and $E_{0}=8 \pi \rho^{2} \gamma^{2} I_{A} /\left(\lambda_{\mathrm{w}} c K A_{J J}\right)$. Simulation of the timedependent effects is performed using the algorithm described in the previous section. When constructing numerical simulation code, it is more convenient to rewrite eq. (4.6) in the following form:

$$
\frac{d^{2} a_{1}^{(j)}}{d \hat{z}^{2}}=i \hat{E}^{(j)}, \quad \frac{d \hat{E}^{(j)}}{d \hat{z}}=a_{1}^{(j)}
$$

To find the evolution of the beam bunching $a_{1}^{(j)}$ and of the electromagnetic field, we should define the initial conditions at the undulator entrance at $\hat{z}=0$. At the entrance of the 
undulator there is no radiation field and the fluctuations of the beam current density caused by shot noise in the electron beam play the role of input signal. At the number of the particles in the bunch equal to $N$, the number of particles per one box is equal to $N_{\lambda}=N / N_{\mathrm{b}}$. To calculate correctly the initial conditions, the following algorithm can be used [18]. One can distribute $N_{\lambda}$ particles randomly in each box. Amplitude and phase of the beam bunching are calculated in accordance with relation:

$$
\begin{aligned}
\left.a_{1}^{(j)}\right|_{\hat{z}=0} & =N_{\lambda}^{-1} \sum_{k=1}^{N_{\lambda}} \exp \left(-i \psi_{k}^{(j)}\right) \\
\left.\frac{d a_{1}^{(j)}}{d \hat{z}}\right|_{\hat{z}=0} & =0
\end{aligned}
$$

where $\psi_{k}^{(j)}=2 \pi s_{k}^{(j)} / \lambda$ is the electron phase and $s_{k}^{(j)}$ is axial coordinate of the particle in the bunch. The initial condition on the derivative of the density modulation is set equal to zero, because the effect of the velocity fluctuations at the undulator entrance can be neglected as we discussed above.

Giving the possibility to simulate actual number of the particle in the beam, such an algorithm for preparing initial conditions requires considerable CPU time. In the program we realized another approach which provides the same results without extra consumption of CPU time. The idea is as follows. The number of particles per box $N_{\lambda}$ is large, typical values are $10^{4} \div 10^{6}$ for the X-ray and VUV FELs. In this case the bunching in each box is the sum of large number of random phasors with fixed amplitudes and uniformly distributed on $(0,2 \pi)$ phases. Using the central limit theorem, we can conclude that phases of bunching parameters are distributed also uniformly and squared modules of amplitudes, $\left|a_{1}\right|^{2}$, are distributed in accordance with the negative exponential distribution:

$$
p\left(\left|a_{1}\right|^{2}\right)=\frac{1}{\left\langle\left|a_{1}\right|^{2}\right\rangle} \exp \left(-\frac{\left|a_{1}\right|^{2}}{\left.<\left|a_{1}\right|^{2}\right\rangle}\right),
$$

where $\left\langle\left|a_{1}\right|^{2}\right\rangle=1 / N_{\lambda}$. The distribution of the modules, $\left|a_{1}\right|$, is the Rayleigh probability density function [19]. So, we use the negative exponential random generator setting $1 / N_{\lambda}$ as mean value to extract the values of $\left|a_{1}\right|^{2}$ for each box and then we extract the square root to find the values of $\left|a_{1}\right|$. The phases of $a_{1}$ are produced by random generator of uniform distribution from 0 to $2 \pi$.

\subsection{Nonlinear simulation code}

Nonlinear time-dependent simulation algorithm is based on similar technique described in the previous sections. We divide the electron beam into $N_{\mathrm{b}}=l_{\mathrm{b}} / \lambda$ boxes. Steady-state FEL equations are used in each box to calculate the motion of the electrons and the 
evolution of the radiation field within one undulator period, i.e. within $\Delta \hat{z}=\lambda_{\mathrm{w}} \Gamma$ (see, e.g. $[21])$ :

$$
\begin{aligned}
& d \hat{P}_{k}^{(j)} / d \hat{z}=-\hat{E}^{(j)} \exp \left(i \psi_{k}^{(j)}\right)+C . C . \\
& d \psi_{k}^{(j)} / d \hat{z}=\hat{P}_{k}^{(j)} \\
& d \hat{E}^{(j)} / d \hat{z}=a_{1}^{(j)}
\end{aligned}
$$

where $j=1, \ldots N_{\mathrm{b}}, k=1, \ldots N_{m}$ and $N_{m}$ is the number of macroparticles in each box and $\hat{P}_{k}^{(j)}=\left(\mathcal{E}_{k}^{(j)}-\mathcal{E}_{0}\right) /\left(\rho \mathcal{E}_{0}\right)$ is the reduced energy deviation from nominal value. The amplitude and the phase of the beam bunching are calculated as follows:

$$
a_{1}^{(j)}=N_{m}^{-1} \sum_{k=1}^{N_{m}} \exp \left(-i \psi_{k}^{(j)}\right)
$$

where $\psi_{k}^{(j)}$ is the electron phase.

To take into account slippage effect, we transfer radiation which interacted with electrons in the $j$ th box into $j+1$ th box after each undulator period.

For the initial shot noise simulations we have used technique proposed in ref. [25]. We choose the number of macroparticles in each box to be equal to $N_{m} \ll N_{\lambda}$. The particles are distributed over phase $\psi$ in the following way:

$$
\psi_{k}^{(j)}=\frac{2 \pi}{N_{m}}\left(k-\frac{1}{2}\right)+\left(2 F_{0}^{1}-1\right) \delta
$$

where $F_{0}^{1}$ is a pseudo-random variable uniformly distributed between 0 and 1 and $\delta=$ $\sqrt{3 N_{m} / N_{\lambda}}$.

It is not evident that such an artificial distribution corresponds to actual initial conditions described above. We performed thorough check of this method and obtained that it is really provides correct statistics, the phases of the bunching, $a_{1}$, are distributed uniformly on $(0,2 \pi)$ and squared modules, $\left|a_{1}\right|^{2}$, are distributed in accordance with eq. (4.9).

\section{Numerical simulations of statistical properties of SASE FEL radiation}

\subsection{Parameters of $70 \mathrm{~nm}$ option of SASE FEL at DESY}

All the numerical results presented in this section refer to the $70 \mathrm{~nm}$ option of SASE FEL at DESY [9] which is the first stage for realization of a full scale facility operating at the wavelength of $6 \mathrm{~nm}[7,8]$.

In the present study we consider the model of rectangular profile of the electron pulse of duration $T=\sqrt{2 \pi} \sigma_{\mathrm{z}} / c \simeq 2$ ps. 
Table 0.1. Parameters of $70 \mathrm{~nm}$ SASE FEL at DESY

\begin{tabular}{ll}
\hline Electron beam & \\
\hline Energy, $\mathcal{E}_{0}$ & $300 \mathrm{MeV}$ \\
Peak current, $I_{0}$ & $500 \mathrm{~A}$ \\
rms bunch length, $\sigma_{\mathrm{z}}$ & $250 \mu \mathrm{m}$ \\
Normalized rms emittance, $\epsilon_{\mathrm{n}}$ & $2 \pi \mathrm{mm} \mathrm{mrad}$ \\
External $\beta$-function, & $100 \mathrm{~cm}$ \\
Number of bunches per train & 7200 \\
Repetition rate & $10 \mathrm{~Hz}$ \\
Undulator & \\
Type & Planar \\
Length of undulator, $L_{\mathrm{w}}$ & $13 \mathrm{~m}$ \\
Period, $\lambda_{\mathrm{w}}$ & $2.73 \mathrm{~cm}$ \\
Peak magnetic field, $H_{\mathrm{w}}$ & $4.97 \mathrm{kGs}$ \\
Radiation & \\
Wavelength, $\lambda$ & $71.4 \mathrm{~nm}$ \\
Bandwidth, $\Delta \lambda / \lambda$ & $1 \%$ \\
Power averaged over pulse & $600 \mathrm{MW}$ \\
Flash energy & $0.1 \mathrm{~mJ}$ \\
Average power & $90 \mathrm{~W}$ \\
\hline
\end{tabular}

\subsection{General remarks}

In this section we present the results of numerical studies of the operation of SASE FEL in the linear and nonlinear regime. Simulations have been performed by means of large number of simulation runs. The result of each run contains parameters of the output radiation (field and phase) stored in the boxes over the full length of the radiation pulse. We performed 2400 statistically independent runs with linear simulation code and 100 runs with nonlinear simulation codes. At the next stage of numerical experiment the arrays of data have been handled with postprocessor codes to extract different statistical properties of the SASE FEL radiation. The first and the second order time correlation functions are calculated using eqs. (2.49) and (2.59). Spectrum of the radiation pulse is calculated by means of Fourier transformation (4.3) of the temporal structure. The first and the second order spectral correlation functions are calculated using eqs. (2.25) and (2.29). Normalized envelope of the radiation spectrum is reconstructed from the first order time correlation function as follows

$$
G(\Delta \omega)=\frac{1}{2 \pi} \int_{-\infty}^{\infty} d \tau g_{1}(\tau) \exp (-i \Delta \omega \tau) .
$$

Different types of statistical distributions have been studied by means of plotting histograms of large number of statistical data.

Parameters for the numerical example correspond to those presented in Table 1. For this numerical example parameter $\rho$ is equal to $4 \times 10^{-3}$, the integration step of the dimensionless equations (4.7), (4.10) and (4.11) is equal to 0.05 and the number of boxes is equal to 8400 . When performing linear simulation, initial shot noise conditions have been simulated with the technique described in section 4 which correctly corresponds to 
actual number of the electrons in the bunch, $N=6 \times 10^{9}$. Nonlinear simulations have been performed using 20 macroparticles per box. Initial shot noise also corresponds to that of actual electron beam. Integration of the self-consistent equations (4.7), (4.10) and (4.11) has been performed by Runge-Kutta scheme. The codes operate relatively fast and provide the possibility to store the required number of statistical data within reasonable time.

To test linear simulation code we used rigorous results of the SASE FEL theory presented in section 2. Nonlinear simulation code has been tested by means of linear simulation code at the linear stage of operation. Such a cross-testing have shown full physical consistency of the results obtained by means of analytical approach and by means of numerical codes.

\subsection{Linear mode of operation}

We begin study the properties of SASE FEL radiation with the high gain linear regime for specific example of the reduced length equal to $\hat{z}=11$. Parameters of the radiation at this point correspond to the exit of the second stage of $70 \mathrm{~nm}$ option SASE FEL at DESY.

Temporal and spectral structure of the radiation pulse. Fig. 5.1 presents typical time structure of the radiation pulse. Fig. 5.2 presents the spectrum of the radiation pulse corresponding to this shot. The time and spectral structure of the radiation pulse changes from shot to shot and the information about the properties of the radiation can be obtained only on the statistical analysis of series of shots. Below we present such analysis of SASE FEL linear mode of operation and compare the results with theoretical predictions of Section 2.

Fluctuations of the radiation power. A brief view on Fig. 5.1 indicates that instant values of the radiation power are random values and practically important problem is to find the law for the probability density function. It has been shown in section 2 that in the linear regime of SASE FEL operation instant values of the radiation power must be distributed in accordance with the negative exponential law. Histogram presented in Fig. 5.3 agrees very well with this prediction.

The first and the second order time correlation functions. In Fig. 5.4 we present the results of calculations of the first and the second order time correlation functions. The circles on these plots are calculated with analytical asymptotical formulae (2.51) and (2.61).

In Fig. 5.5 we present the plot of the real and imaginary parts of the first order time correlation function. It is seen that at finite undulator length there exists nonzero imaginary part of the first order correlation function. The real part is close with graphical accuracy to the asymptotical value. 

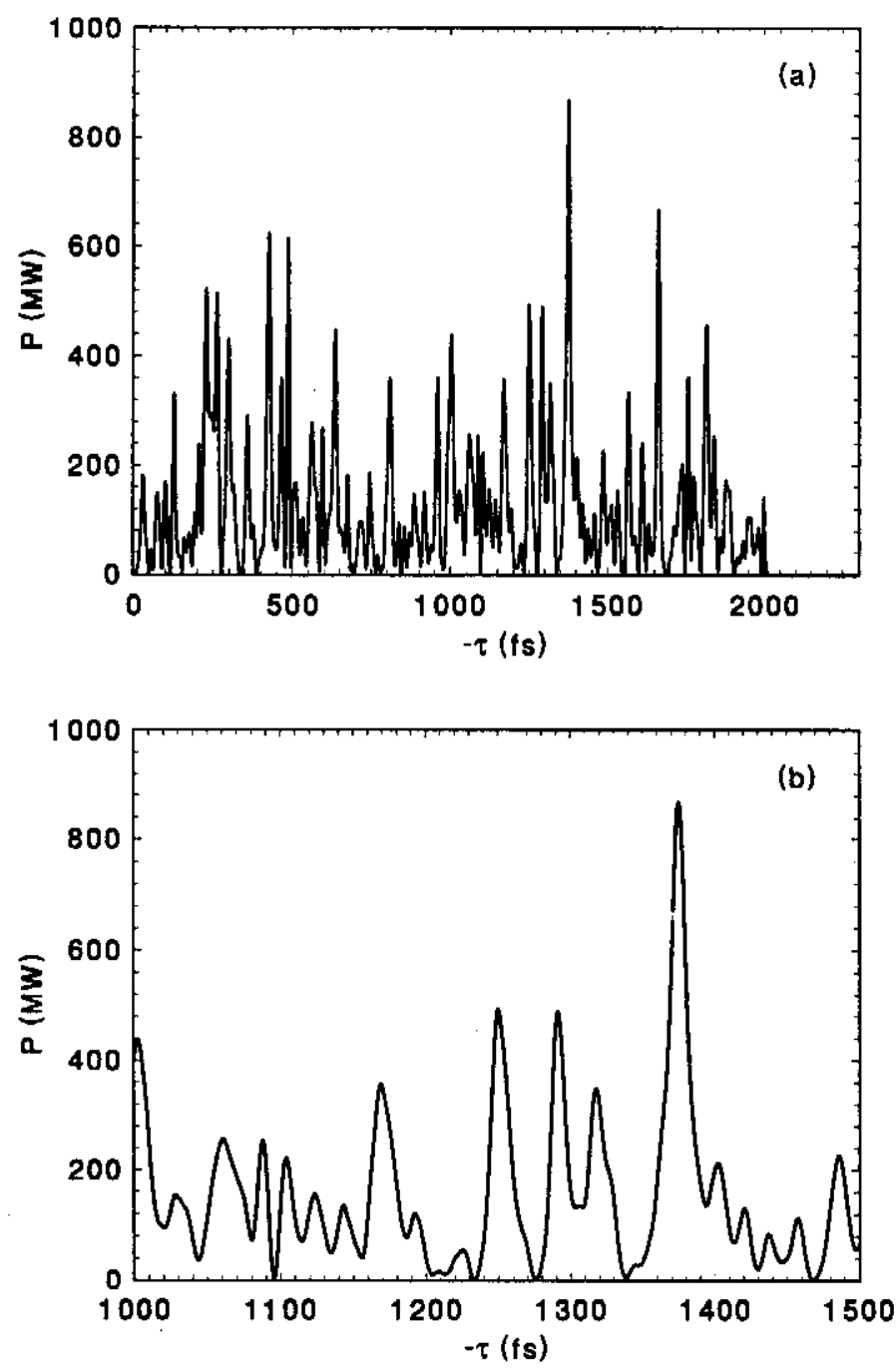

Fig. 5.1. Typical temporal structure of the radiation pulse (one shot) at the reduced length of the FEL amplifier $\hat{z}=11$. Graph (a) is plotted over the full length of the radiation pulse and graph (b) presents enlarged fraction of graph (a). Calculations have been performed with linear simulation code. 

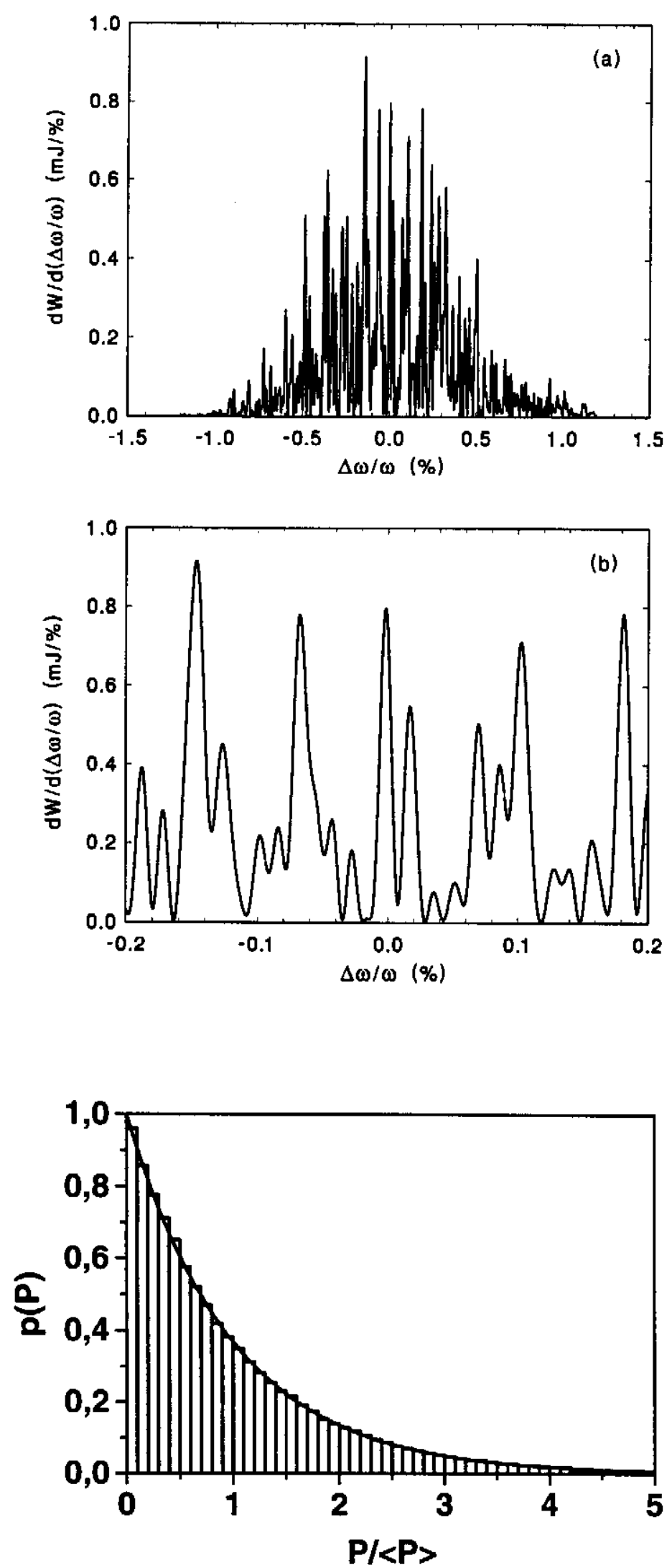

Fig. 5.2. Typical spectrum of the radiation pulse (one shot) at the reduced length of the FEL amplifier $\hat{z}=11$. Graph (a) is plotted over the full spectrum width and graph (b) presents enlarged fraction of graph (a). Calculations have been performed with linear simulation code.
Fig. 5.3. A histogram of probability density distribution, $p(P)$, of instant output power at the reduced length of the FEL amplifier $\hat{z}=11$. Calculations have been performed with linear simulation code over $2 \times 10^{5}$ independent statistical events (see Fig. 5.1). $\langle P\rangle$ denotes the average power. The solid curve represents the negative exponential distribution $p(P)=$ $\exp (-P /\langle P\rangle)$. 

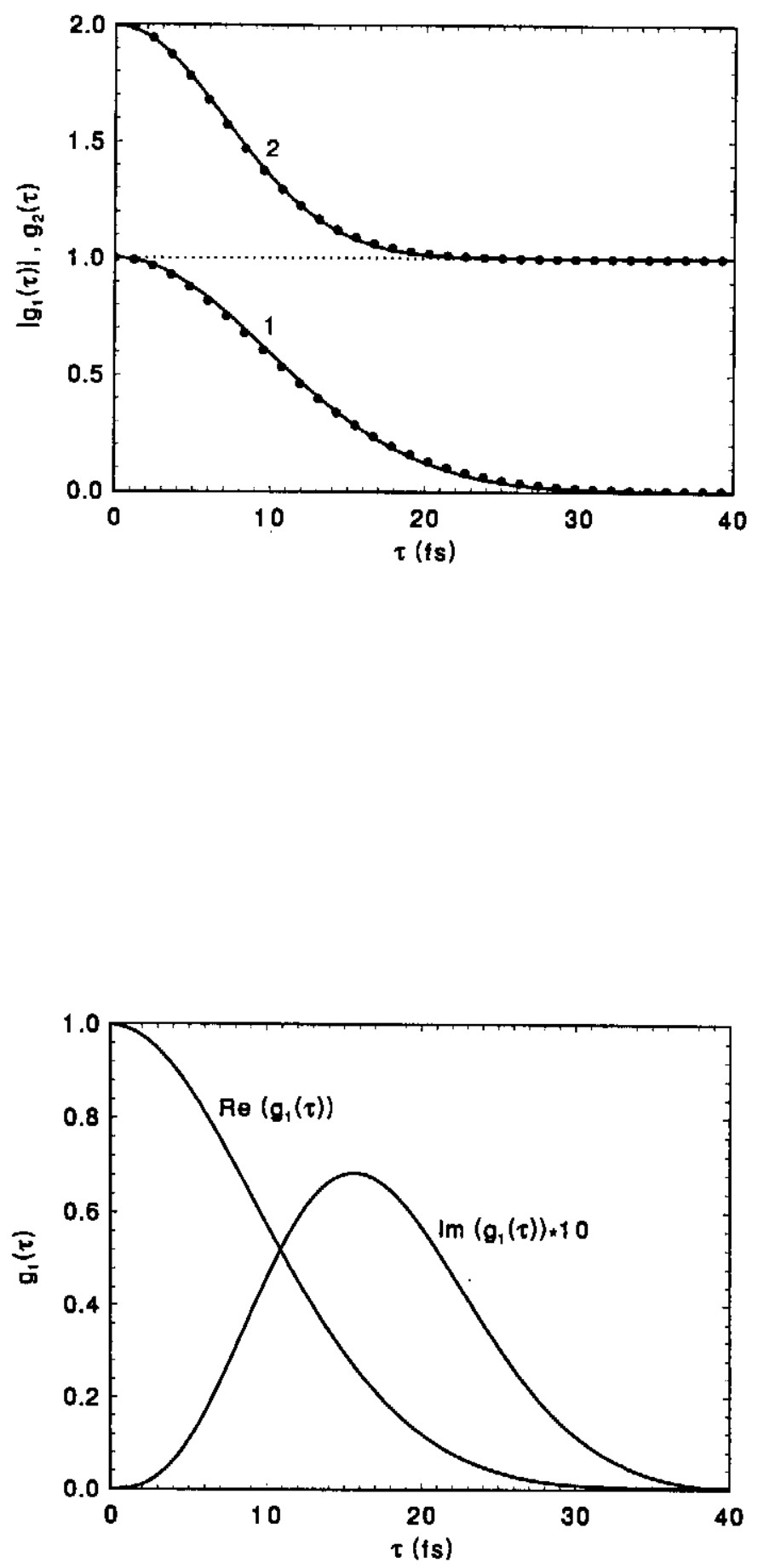

Fig. 5.4. Module of the first order time correlation function, $\left|g_{1}(\tau)\right|$ (curve1), and the second order time correlation function, $g_{2}(\tau)$ (curve 2), of the radiation pulse at the reduced length of the FEL amplifier $\hat{z}=11$. Solid curves are the results of calculations with analytical formulae (2.51) and (2.61). Circles are the result of calculations with linear simulation code over $5 \times 10^{4}$ independent statistical events.

Fig. 5.5. Real and imaginary parts of the first order time correlation function $g_{1}(\tau)$ of the radiation pulse at the reduced length of the FEL amplifier $\hat{z}=11$. Calculations have been performed with linear simulation code over $5 \times 10^{4}$ independent statistical events. 
The spectrum of SASE FEL. Visual analysis of the spectrum of the radiation pulse for several shots (see Fig. 5.2) indicates that spikes in the spectrum are approximately inside some envelope. The straightforward way to obtain this envelope is to average large number of spectrum data. In Fig. 5.6 we present spectrum of SASE FEL radiation averaged over 2400 shots. It is seen that even at such a number of independent runs the accuracy of the spectrum reconstruction is not excellent and relative error in each point of spectrum is still visible.
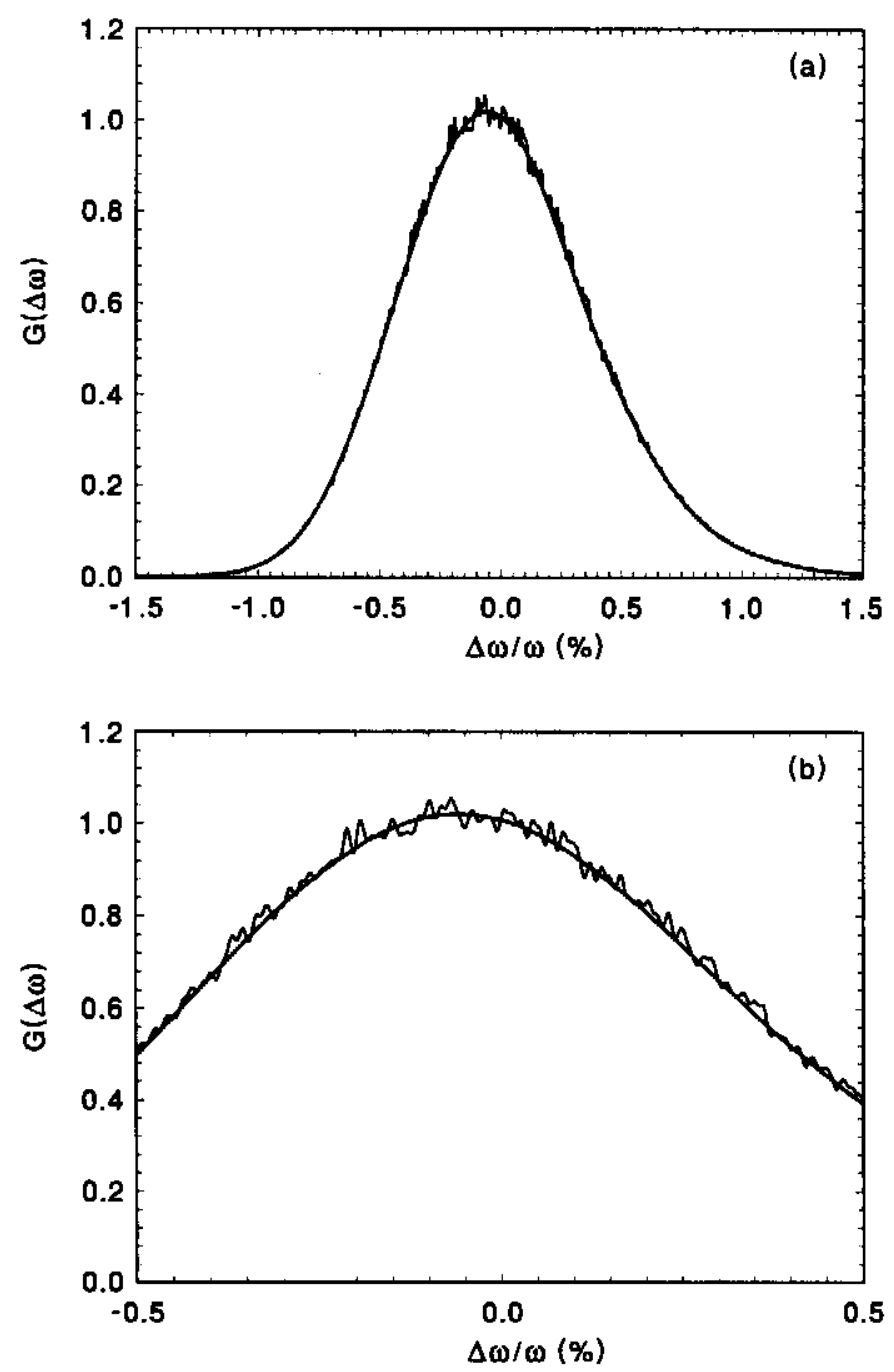

Fig. 5.6. Normalized spectrum of the radiation from SASE FEL, $G(\Delta \omega)$, at the reduced length of the FEL amplifier $\hat{z}=$ 11. Averaging has been performed over 2400 shots. Smooth curve is the result of spectrum reconstruction using the first order time correlation function (see Fig. 5.5 and eq. (5.1)). Graph (a) is calculated over full spectrum and graph (b) is enlarged fraction of graph (a).

Another technique for obtaining the envelope of spectrum of SASE FEL consists in its reconstruction from the first order time correlation function (5.1). Analysis of the plot presented in Fig. 5.4 indicates that the first order correlation function is calculated with much higher accuracy. This is connected with the fact that the statistics for the correlation function calculations is higher by a factor of the number of spikes in the bunch. In Fig. 5.5 we present the real and imaginary parts of the first order time correlation function. These 


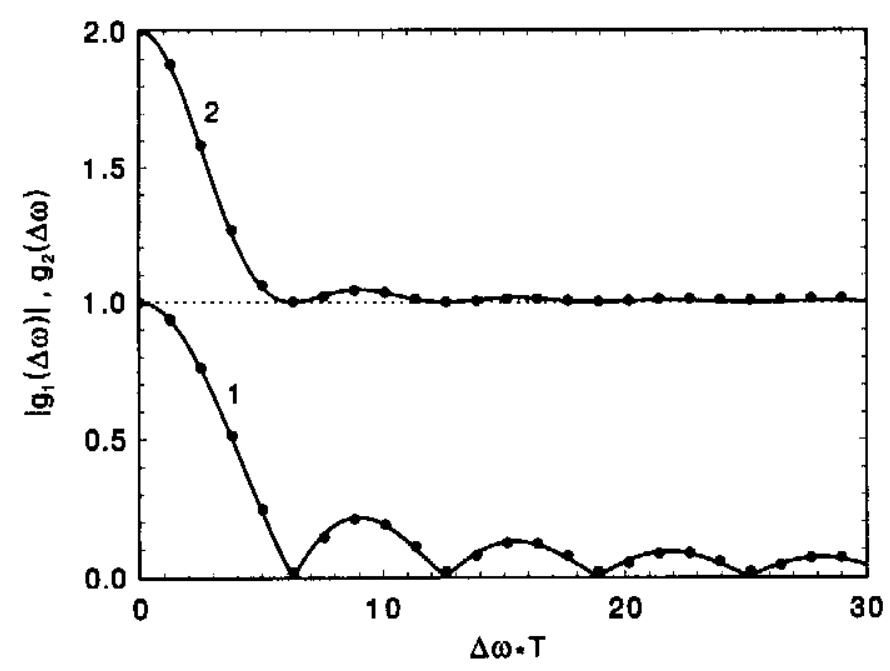

Fig. 5.7. Module of the first order spectral correlation function, $\left|g_{1}(\Delta \omega)\right|$ (curve1), and the second order spectral correlation function, $g_{2}(\Delta \omega)$ (curve 2), of the radiation pulse at the reduced length of the FEL amplifier $\hat{z}=11$. Solid curves are the results of calculations with analytical formulae (2.26) and (2.31). Circles are the result of calculations with linear simulation code over $5 \times 10^{4}$ independent statistical events.

data have been used for the spectrum reconstruction (solid line in Fig. 5.6). It is seen that such a technique provides very precise results. Our experience have shown that this technique provides almost perfect reconstruction of the spectrum at the number of runs of several tens. Analysis of the spectrum presented in Fig. 5.6 shows that its shape and position of the maximum only slightly differ from those predicted by analytical asymptotic formula (2.18). This difference is connected mainly with nonzero imaginary part of the first order time correlation function at finite undulator length (see Fig. 5.5).

The first and the second order spectral correlation functions. At large number of statistically independent runs there is the possibility to perform precise calculations of the first and the second order spectral correlation functions. Fig. 5.7 presents the results of the calculations of these functions. It is seen that there is excellent agreement between numerical and analytical results. In principle, this numerical results model actual experimental situation and give the answer on the required statistics. The results of spectral correlation measurements can be used as a tool for measurements of the radiation bunch profile [26]. In the modelled situation the bunch profile was a rectangular one which defined the shape of correlation functions (see eqs. (2.26) and (2.31)).

Fluctuations of the energy in the radiation pulse. We performed numerical study of the fluctuations of the energy in the radiation pulse integrated over finite time. In accordance with theoretical predictions the probability density function of the energy can be described rather well with the gamma distribution (2.43) which is general feature of completely chaotic polarized light. In Figs. 5.8 and 5.9 we present series of histograms calculated with 2400 independent shots. The value of parameter $M$ reconstructed from simulated distributions is close with an accuracy of about one per cent to theoretical values given by relation (2.62). Analysis of these histograms shows that within statistical accuracy the 

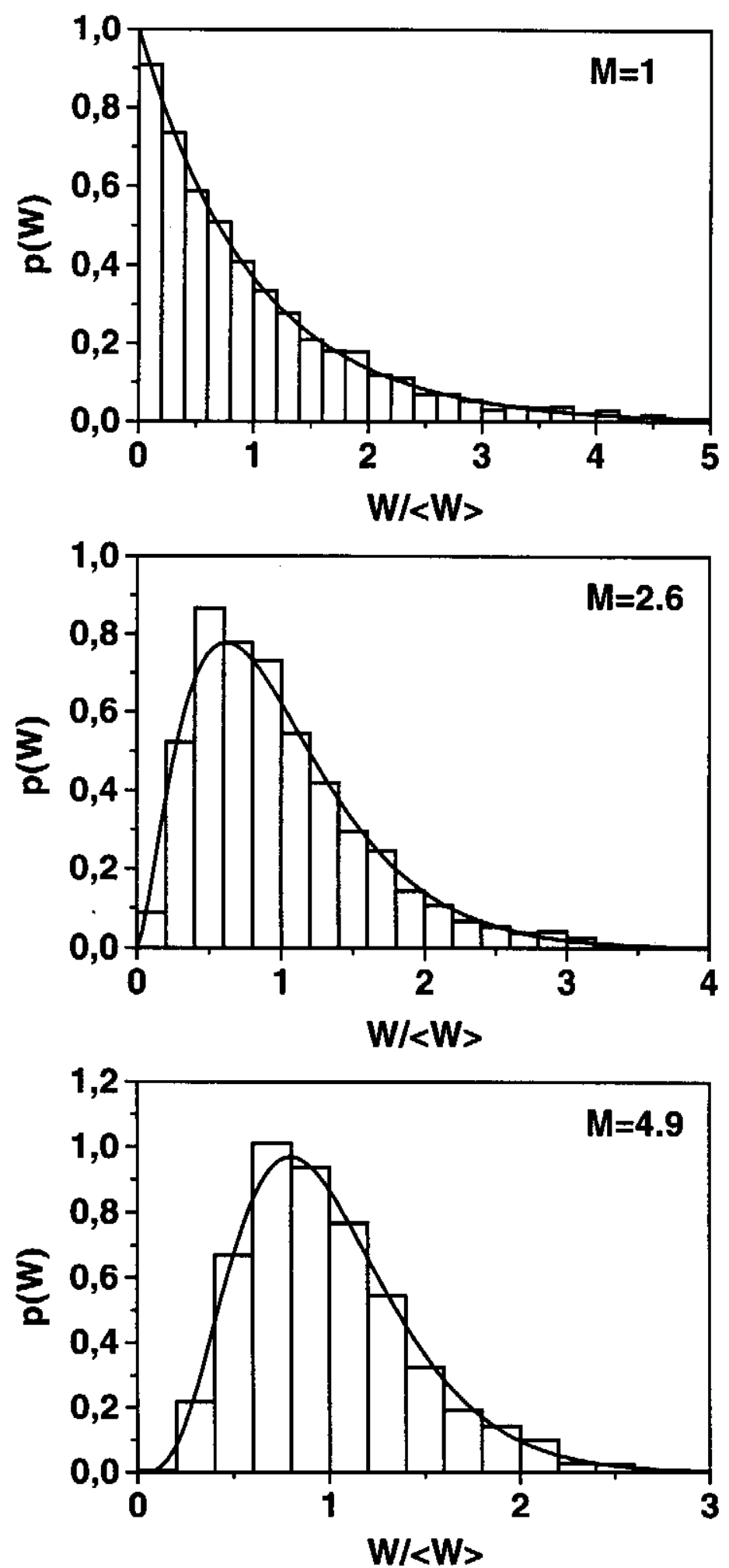

Fig. 5.8. Histograms of the probability density distribution, $p(W)$, of the radiation energy $W$ detected during time $\delta T$. The reduced length of the FEL amplifier is equal to $\hat{z}=11$. Calculations have been performed with linear simulation code over 2400 shots (see Fig. 5.1). Measurement time interval $\delta T$ and parameter $M$ are connected by relation (2.62) (see also Fig. 5.10). $\langle W\rangle$ denotes the average energy. The solid curves represent gamma distribution $(2,43)$. 

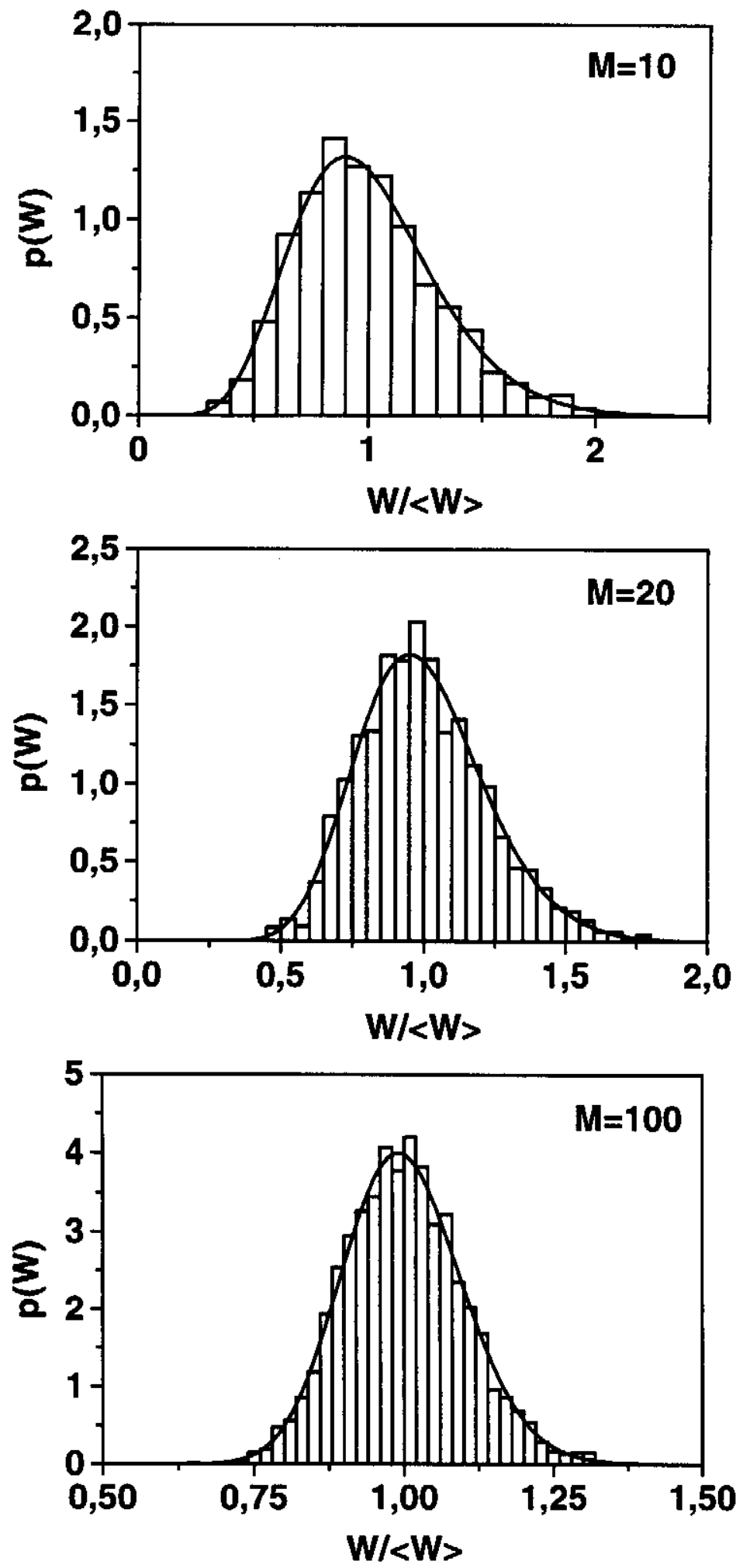

Fig. 5.9. Histograms of the probability density distribution, $p(W)$, of the radiation energy $W$ detected during time $\delta T$. The reduced length of the FEL amplifier is equal to $\hat{z}=11$. Calculations have been performed with linear simulation code over 2400 shots (see Fig. 5.1). Measurement time interval $\delta T$ and parameter $M$ are connected by relation (2.62) (see also Fig. 5.10). $\langle W\rangle$ denotes the average energy. The solid curves represent gamma distribution (2.43). 
gamma distribution describes rather well the probability distribution of the energy in the radiation pulse.

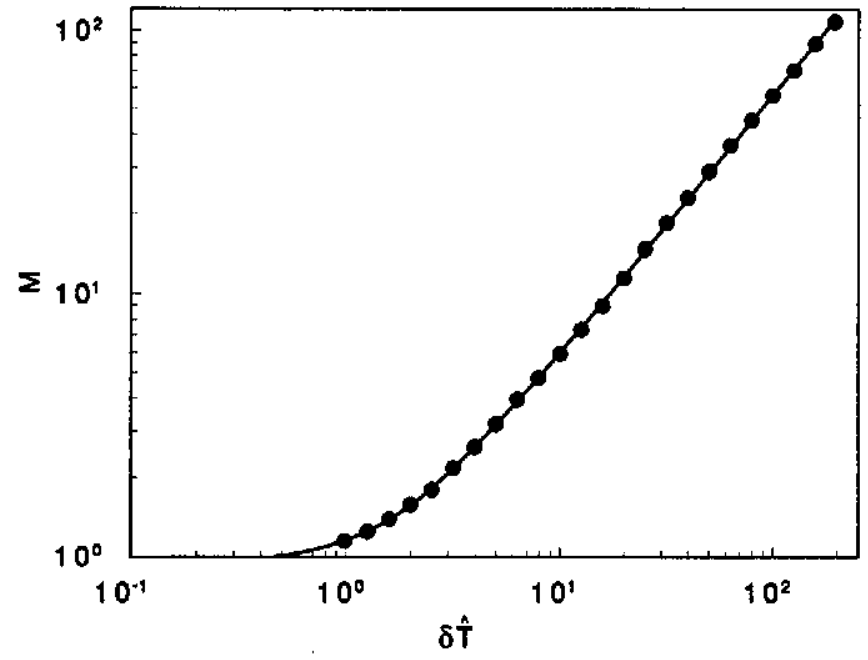

Fig. 5.10. The dependence of the parameter $M$ on the value of the time interval $\delta \hat{T}=\delta T \sigma_{A} . \mathrm{FEL}$ amplifier operates in the linear regime at the reduced length of the FEL amplifier $\hat{z}=11$. Solid curve is the result of calculations with analytical formula (2.62). Circles are the result of calculations with linear simulation code over 2400 shots.

Fluctuations of the radiation energy at the exit of the monochromator. For a number of planned experiments a monochromator should be installed at the exit of SASE FEL and the problem arises about the shot-to-shot distribution of the radiation energy after the monochromator. Theoretical analysis predicts that energy at the exit of the monochromator will be approximately distributed in accordance with the gamma distribution (2.43). Parameter $M=1 / \sigma_{W}^{2}$ of the distribution, is given by formulae (2.39) and (2.38) for the Gaussian and rectangular monochromator line, respectively. Figs. 5.11 and 5.12 show good agreement between the analytical and numerical calculations of the parameter of the gamma distribution, $M$. Figs. 5.13 and 5.14 show that gamma distribution fits rather well the numerical data. It is important to notice that the gamma distribution describes the fluctuations of the energy after monochromator with any arbitrary line shape. Parameter of the distribution, $M$, should be calculated numerically in this case.

Effective power of shot noise. In the high gain linear regime the output radiation power of SASE FEL grows exponentially along the undulator length. The radiation power $\left\langle P_{\text {out }}\right\rangle=$ $\langle W\rangle / T$ averaged over an ensemble is equal to $[12,13,14]$ :

$$
<P_{\text {out }}>=\rho P_{\mathrm{b}} \frac{\sqrt{4 \pi} \rho}{3 \sqrt{\sqrt{3} \hat{z}} N_{\lambda}} \exp [\sqrt{3} \hat{z}],
$$

where $P_{\mathrm{b}}=\gamma m c^{2} I_{0} / e$ is the electron beam power, $\hat{z}=\Gamma z$ and $N_{\lambda}=2 \pi I_{0} / e \omega_{0}$.

When simulating SASE FEL with steady-state codes, the notion of "effective" power of input signal is usually introduced. The output power of the stead-state high gain FEL amplifier changes exponentially with the undulator length (see, e.g. ref. [21]): 

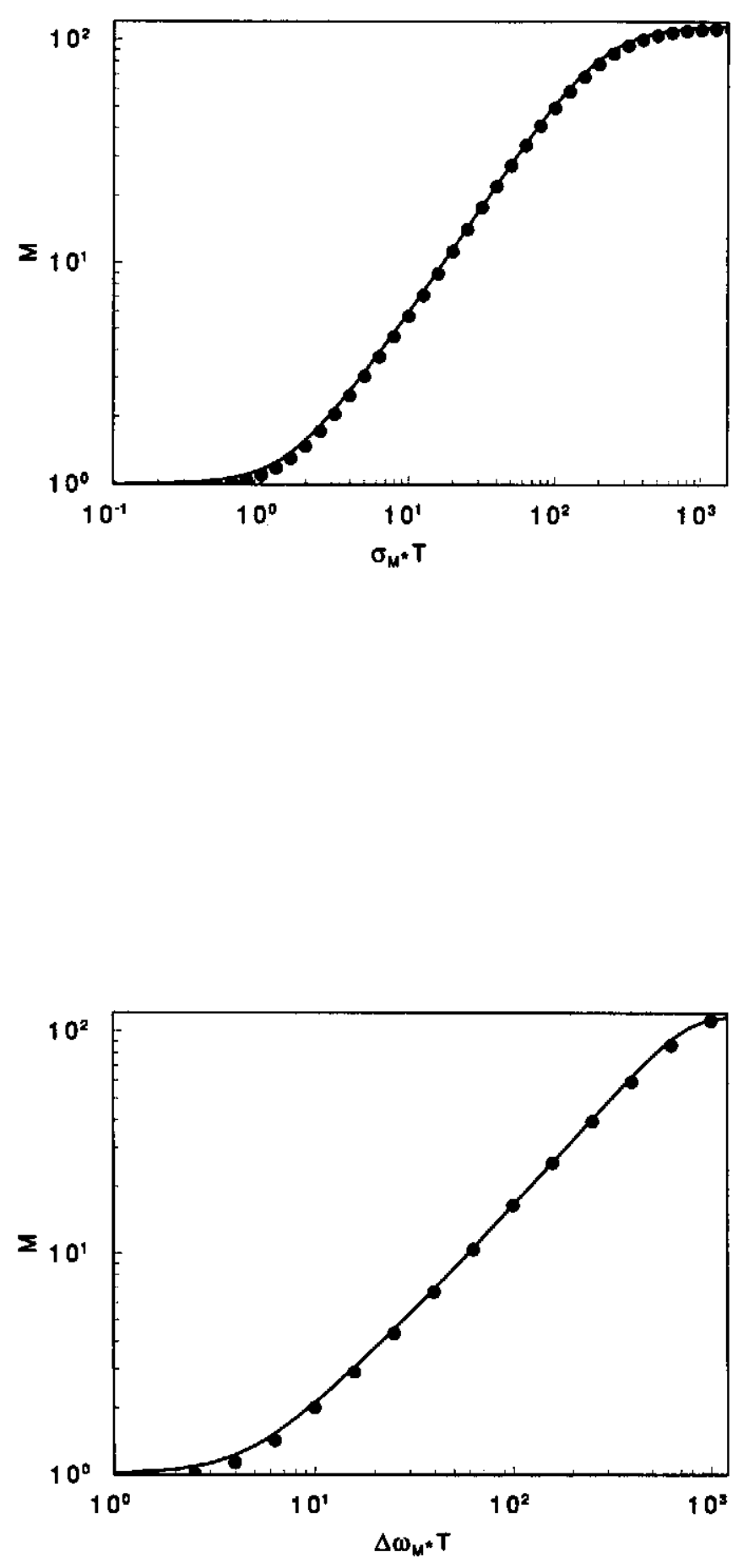

Fig. 5.11. The dependence of the parameter $M$ on the value of the width of the monochromator, $\sigma_{M}$, with Gaussian profile. FEL amplifier operates in the linear regime at the reduced length of the FEL amplifier $\hat{z}=11$. Solid curve is the result of calculations with analytical formula (2.39). Circles are the result of calculations with linear simulation code over 2400 shots.
Fig. 5.12. The dependence of the parameter $M$ on the value of the width of the monochromator $\Delta \omega_{\mathrm{M}}$ with rectangular profile. FEL amplifier operates in the linear regime at the reduced length of the FEL amplifier $\hat{z}=11$. Solid curve is the result of calculations with analytical formula (2.38). Circles are the result of calculations with linear simulation code over 2400 shots. 

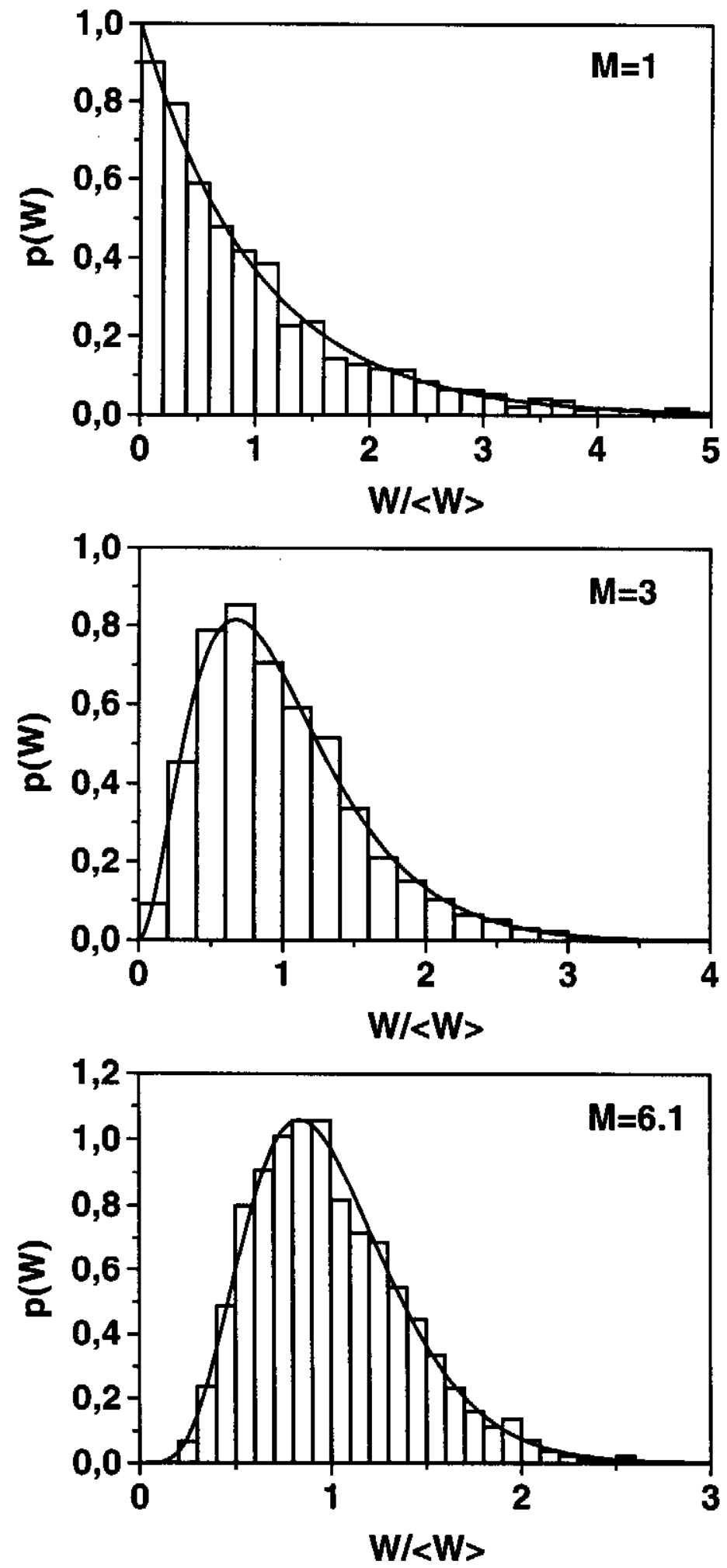

Fig. 5.13. Histograms of the probability density distribution, $p(W)$, of the radiation energy after monochromator for different values of parameter $M$. Calculations have been performed with linear simulation code over 2400 shots (see Fig. 5.2). $\langle W\rangle$ denotes the average energy. The solid curves represent the gamma distribution (2.43) where values of $M$ have been calculated with formula (2.44) (see also Figs. 5.11 and 5.12). 

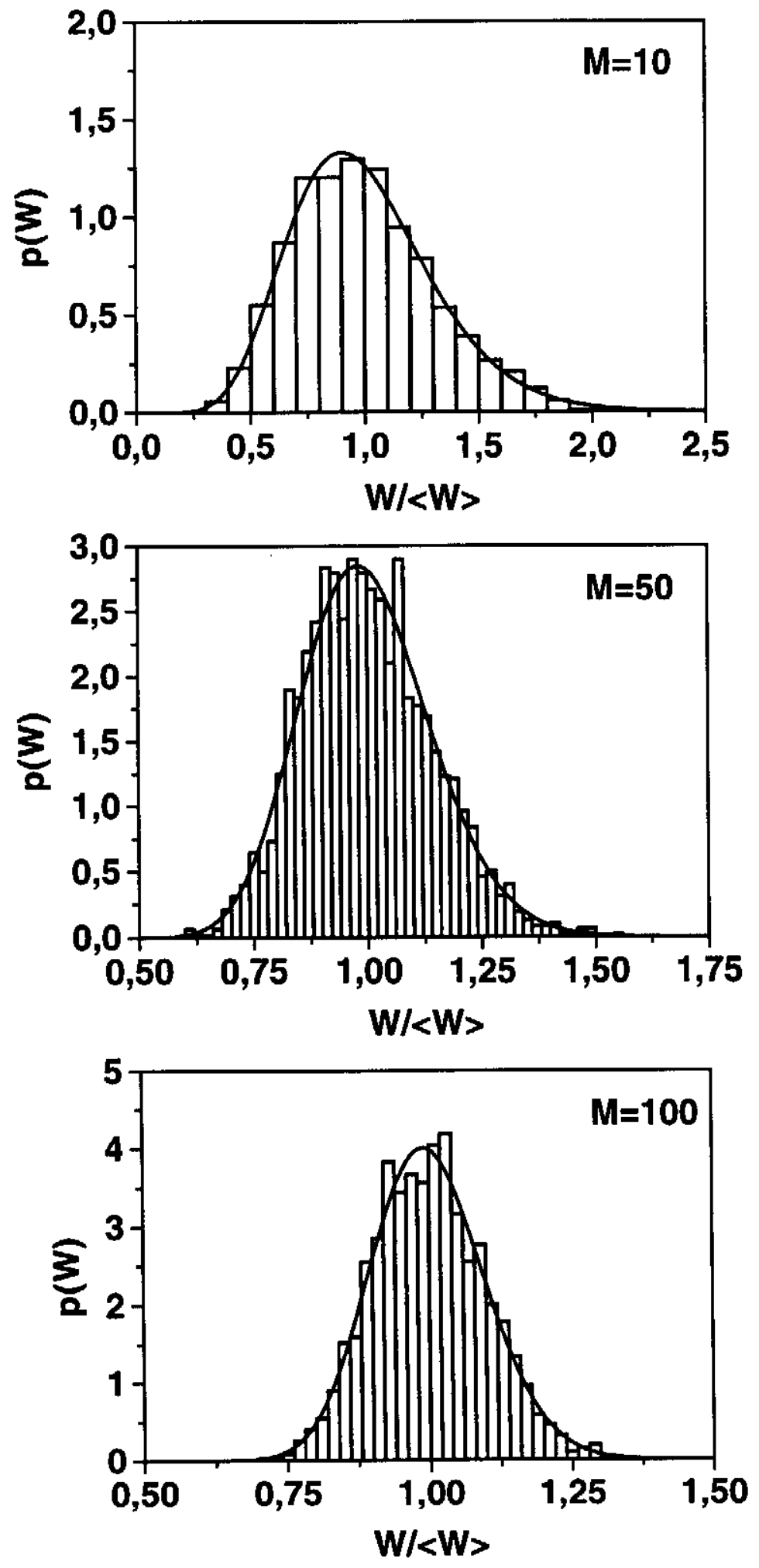

Fig. 5.14. Histograms of the probability density distribution, $p(W)$, of the radiation energy after monochromator for different values of parameter $M$. Calculations have been performed with linear simulation code over 2400 shots (see Fig. 5.2). $\langle W\rangle$ denotes the average energy. The solid curves represent the gamma distribution (2.43) where values of $M$ have been calculated with formula (2.44) (see also Figs. 5.11 and 5.12). 

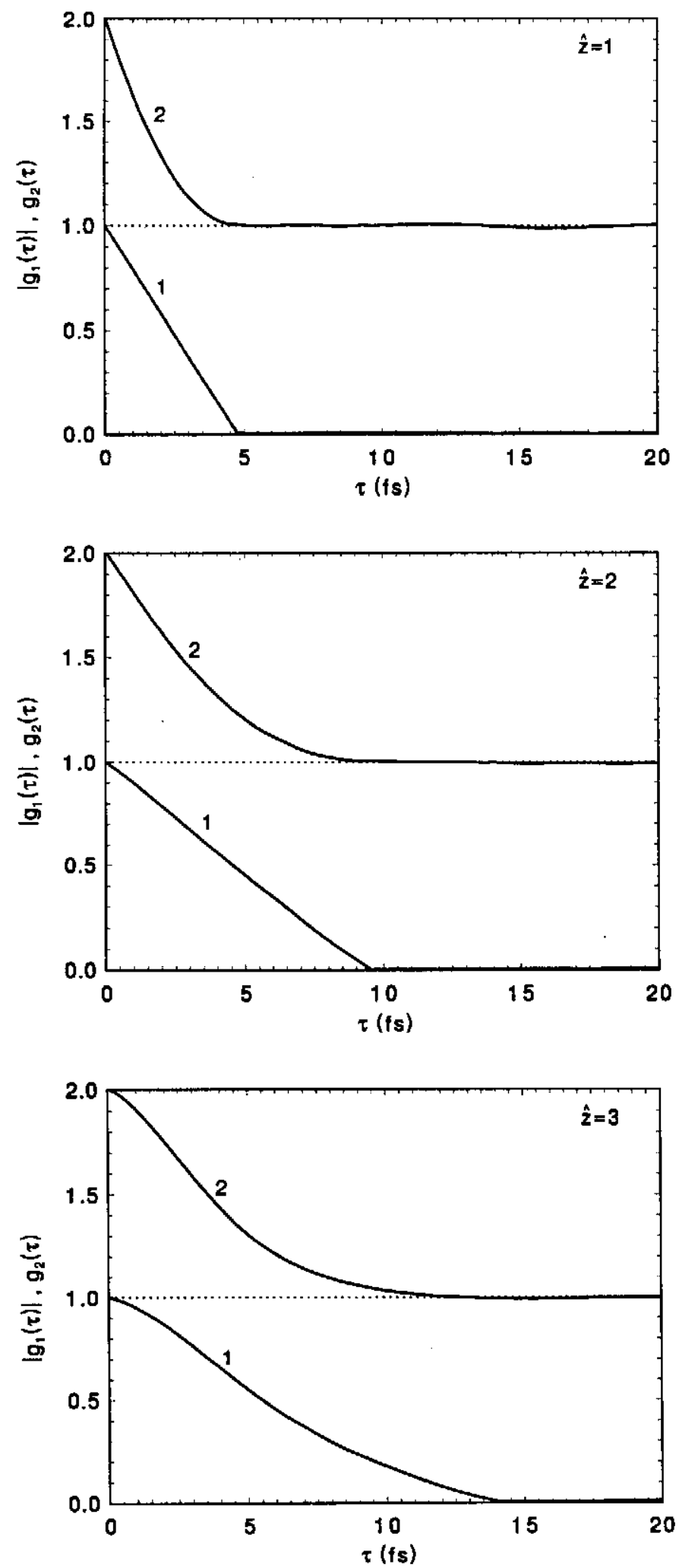

Fig. 5.15. The first and the second order time correlation functions, $g_{1}(\tau)$ (curve1) and $g_{2}(\tau)$ (curve 2), of the radiation pulse at different reduced length of the FEL amplifier. Calculations have been performed with linear simulation code over $10^{4}$ independent statistical events. 

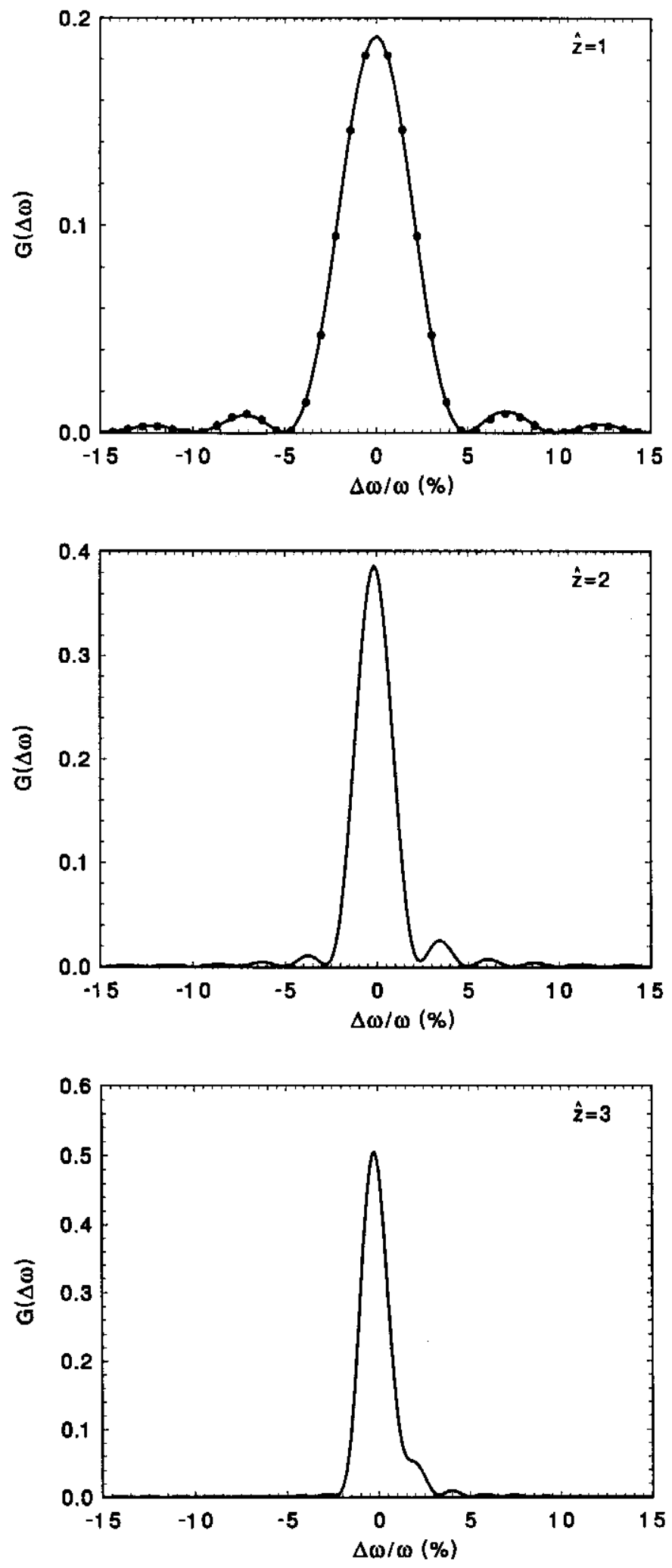

Fig. 5.16. Normalized spectrum of the radiation from SASE FEL $G(\Delta \omega)$ at different reduced length of the FEL amplifier. Spectrum has been reconstructed from the first order time correlation function (see Fig. 5.15 and eq. (5.1)). Circles correspond to normalized spectrum of the undulator radiation. 

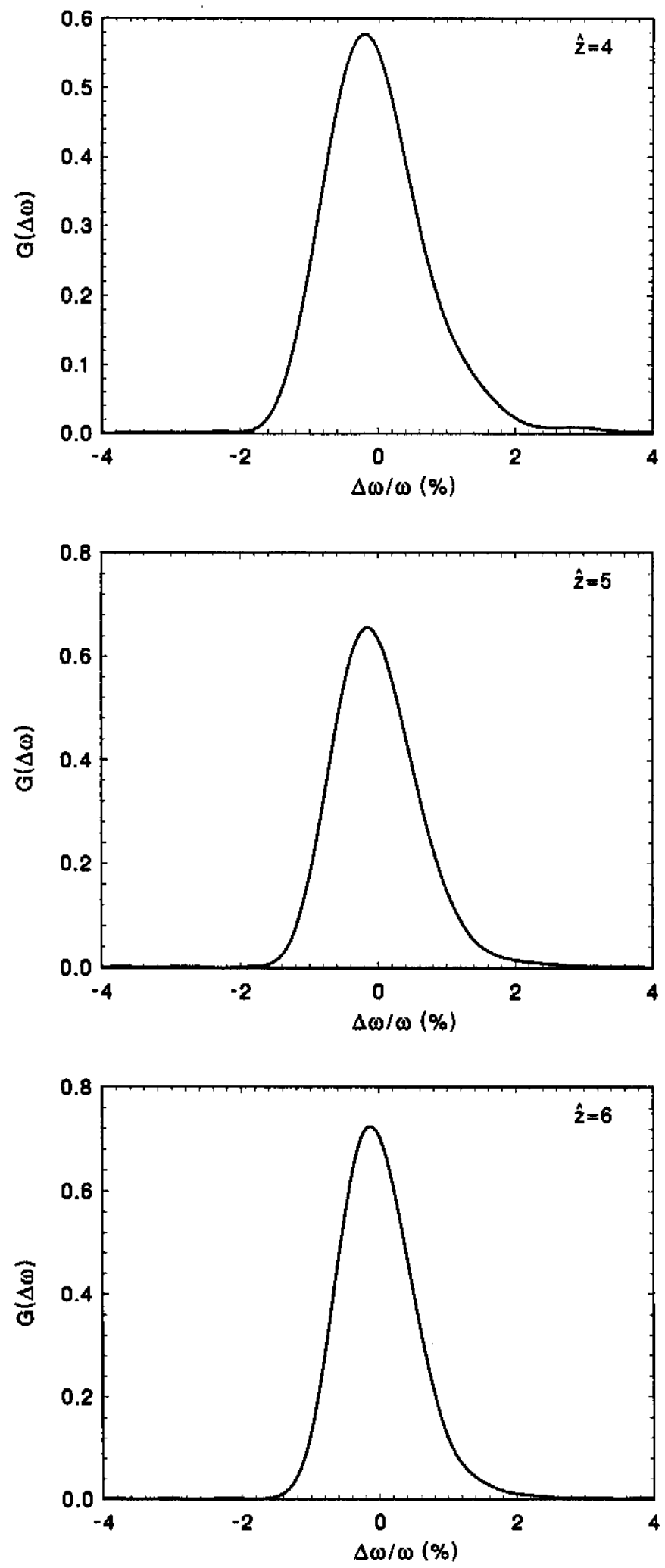

Fig. 5.17. Normalized spectrum of the radiation from SASE FEL $G(\Delta \omega)$ at different reduced length of the FEL amplifier. Spectrum has been reconstructed from the first order time correlation function (5.1). 


$$
P_{\text {out }}=\frac{1}{9} P_{\text {in }} \exp (\sqrt{3} \hat{z})
$$

where $P_{\text {in }}$ is the input power. Combining equations (5.2) and (5.3) we can write the following formula for the effective power of the shot noise, $P_{\mathrm{sh}}$ :

$$
P_{\text {sh }}=P_{\text {in }}=\frac{3^{3 / 4} \rho^{2} P_{\mathrm{b}}}{N_{\lambda}} \sqrt{\frac{4 \pi}{\hat{z}}}
$$

In the considered example the reduced length of the undulator is equal to $\hat{z}=11, \rho=$ $4 \times 10^{-3}$, the number of electrons per wavelength is equal to $N_{\lambda}=7.44 \times 10^{5}$ and the value of effective power of shot noise is equal to $P_{\mathrm{sh}} \simeq 7.9 \mathrm{~W}$.

The value of $P_{\text {sh }}$ can be also obtained from numerical simulations. In the presented numerical example the output power averaged over 2400 shots is equal to $\left\langle P_{\text {out }}\right\rangle \simeq$ 141.3 MW which corresponds to the value of the effective power of shot noise $P_{\mathrm{sh}} \simeq 8 \mathrm{~W}$ (see eq. (5.3)). We see that there is good agreement of analytical and numerical results.

Formation of the amplification process in SASE FEL. In conclusion to this section we present the study of the amplification process formation in SASE FEL. Simulations have been performed with nonlinear simulation code with 100 shots. Temporal structures of the radiation pulses have been used for calculations of the time correlation functions (see Fig. 5.15). Then the radiation spectrums have been reconstructed using Fourier transformation of the first order time correlation function. Figures 5.16 and 5.17 show the evolution of the radiation spectrum with the step equal to one field gain length. One can see that after the first gain length the spectrum of the SASE FEL is identical to the spectrum of the undulator radiation. After several gain lengths the spectrum shape transforms to usual gain curve of the high gain FEL amplifier.

\subsection{Nonlinear mode of operation}

Nonlinear mode of SASE FEL operation has been studied by means of nonlinear simulation code described in section 4. Parameters of the numerical example correspond to those presented in Table 1. The number of shots is equal to one hundred.

Temporal and spectral structure of the radiation pulse. Fig. 5.18 presents typical time structure of the radiation pulse of the FEL amplifier operating in nonlinear regime at the reduced length of the undulator $\hat{z}=20$. Fig. 5.19 presents the spectrum of the radiation pulse corresponding to this shot.

Using statistical technique described above, in this section we study statistical properties of nonlinear mode of operation of SASE FEL. 

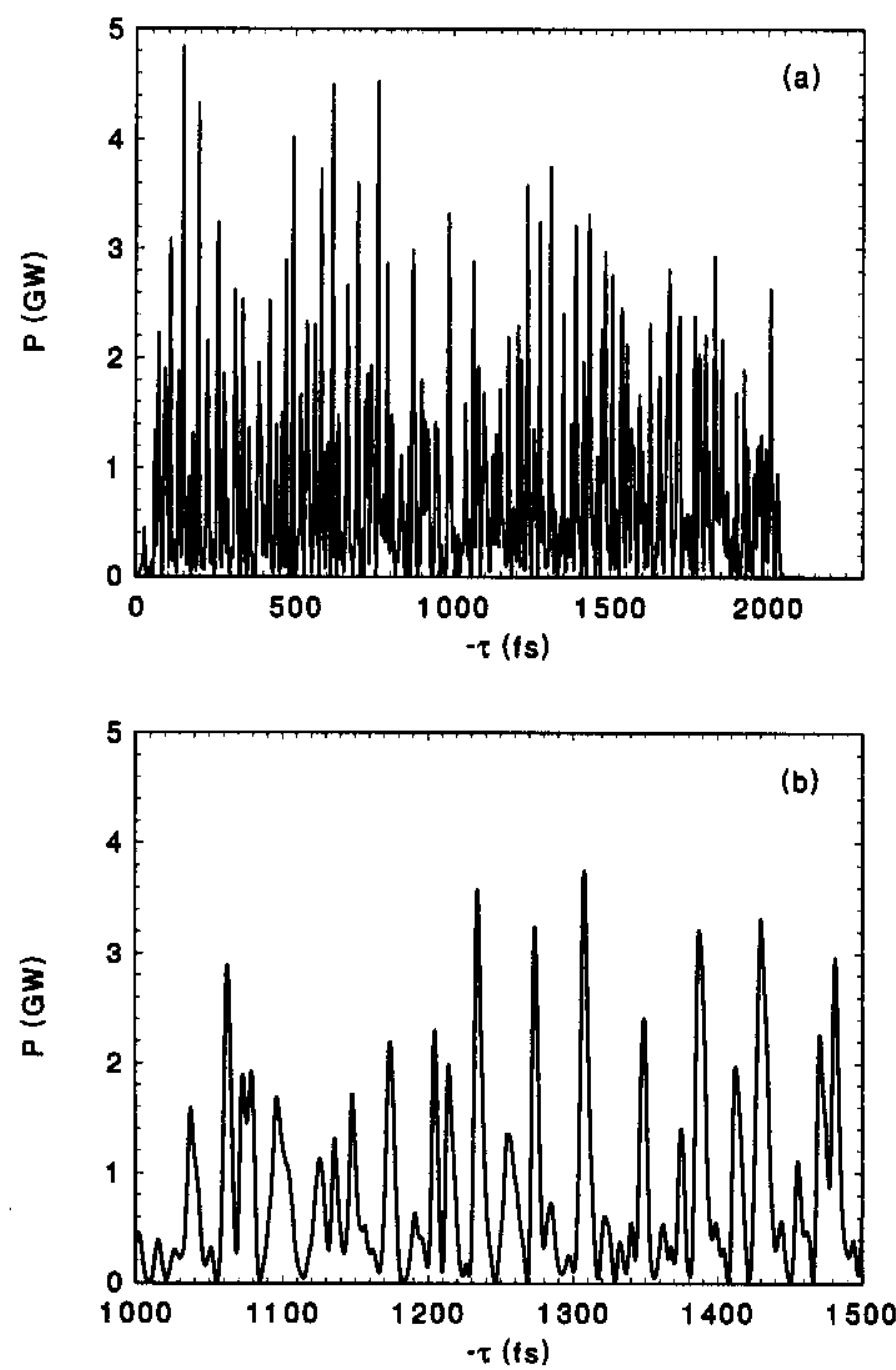

Fig. 5.18. Typical temporal structure of the radiation pulse (one shot) at the reduced length of the FEL amplifier $\hat{z}=20$. Graph (a) is plotted over the full length of the radiation pulse and graph (b) presents enlarged fraction of graph (a). Calculations have been performed with nonlinear simulation code. 

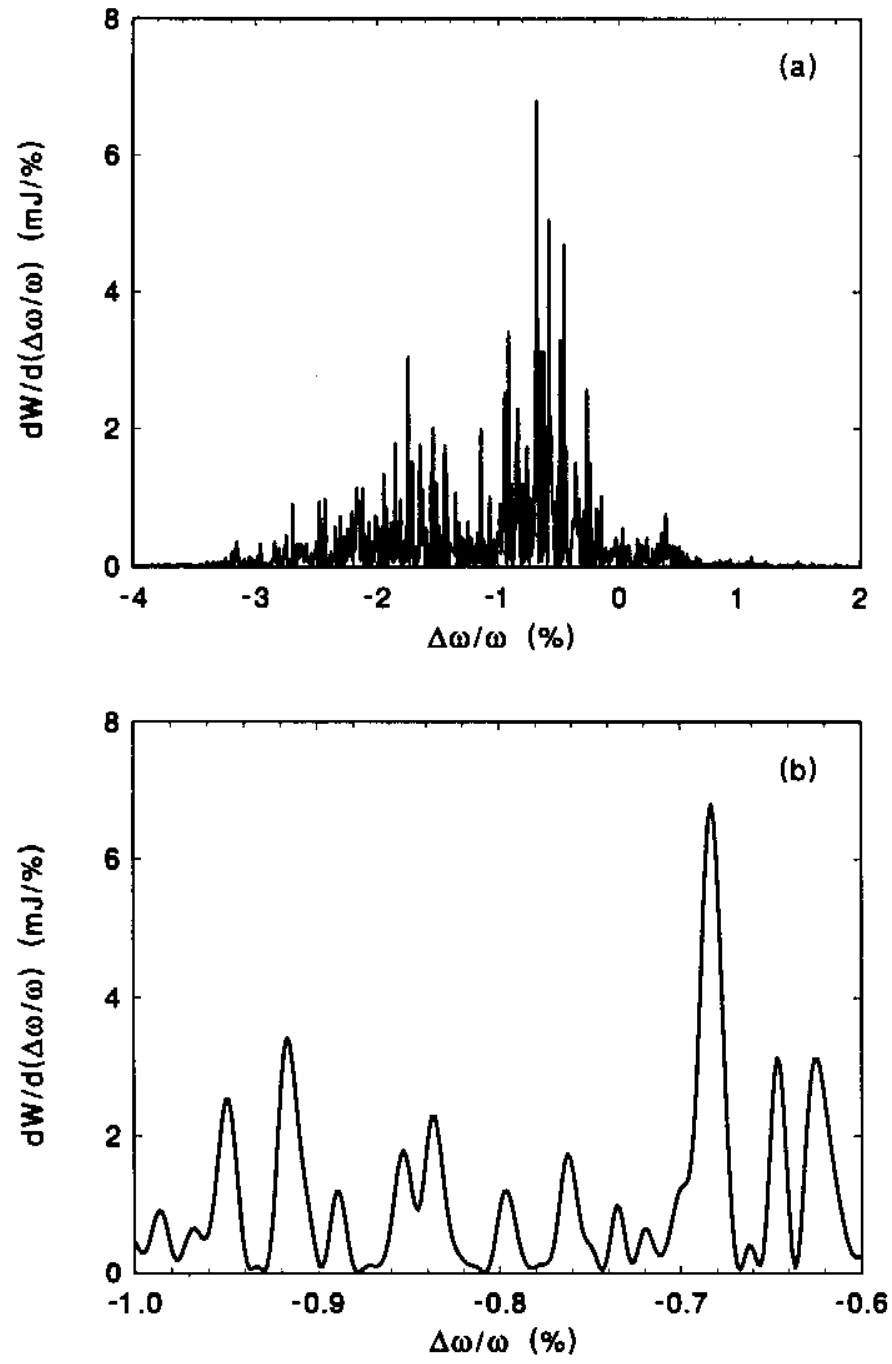

Fig. 5.19. Typical spectrum of the radiation pulse (one shot) at the reduced length of the FEL amplifier $\hat{z}=20$. Graph (a) is plotted over the full spectrum width and graph (b) presents enlarged fraction of graph (a). Calculations have been performed with nonlinear simulation code. 
Average output power. In Fig. 5.20 we present the dependence of the average power of SASE FEL on the reduced length ${ }^{2}$. For comparison we also present the plot for the output power for the steady-state FEL amplifier starting with the value of input power of $8 \mathrm{~W}$ (corresponding to the value of effective power of shot noise).

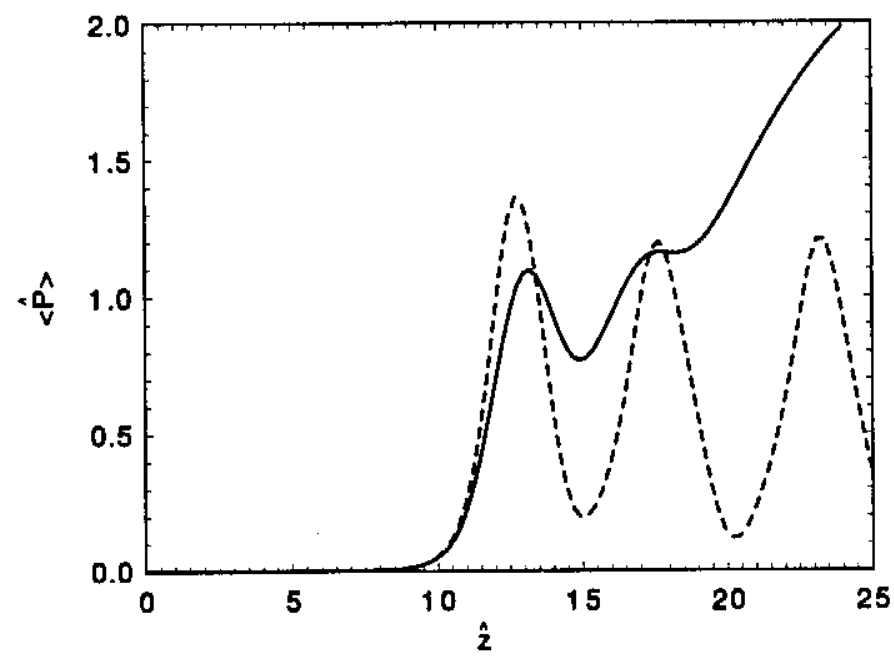

Fig. 5.20. Average power of the radiation from SASE FEL, $\langle\hat{P}>$, as function of the reduced length of the FEL amplifier. Calculations have been performed with nonlinear simulation code over $10^{4}$ independent events. The dotted curve presents the output power of the FEL amplifier operating in the steady-state regime.

It is seen that the main difference occurs at the nonlinear stage of amplification. In the case of the FEL amplifier with external seeding the output power reaches its maximal value at the saturation point, while the output power of SASE FEL begins to grow. Saturation effect in the steady-state FEL amplifier is defined by the fact that the bunched beam effectively interacts with the electromagnetic wave along the length which is of the order of the gain length $l_{\mathrm{g}} \sim \Gamma^{-1}$. At this stage of amplification electrons lose a visual fraction of their energy which results in the violation of the resonance condition. As a result, the beam is overmodulated, the most fraction of electrons fall into the accelerating phase of effective potential and the electron beam becomes to take off the power from the electromagnetic wave.

In the case of SASE FEL the process of the field growth does not stop at saturation point.

The first and the second order time correlation functions. In Fig. 5.21 we present the results of calculations of the first and the second order time correlation functions. It is seen that there is significant difference with respect to the linear mode of operation (see Fig. 5.4). First, we obtain that relation $g_{2}(\tau)=1+\left|g_{1}(\tau)\right|^{2}$ does not takes place in the nonlinear regime. Second, the first order time correlation function begins to have two maxima at the increase of the undulator length.

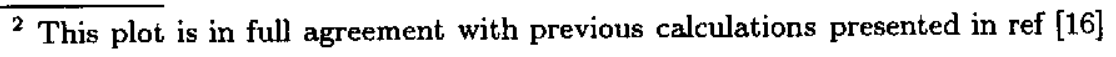



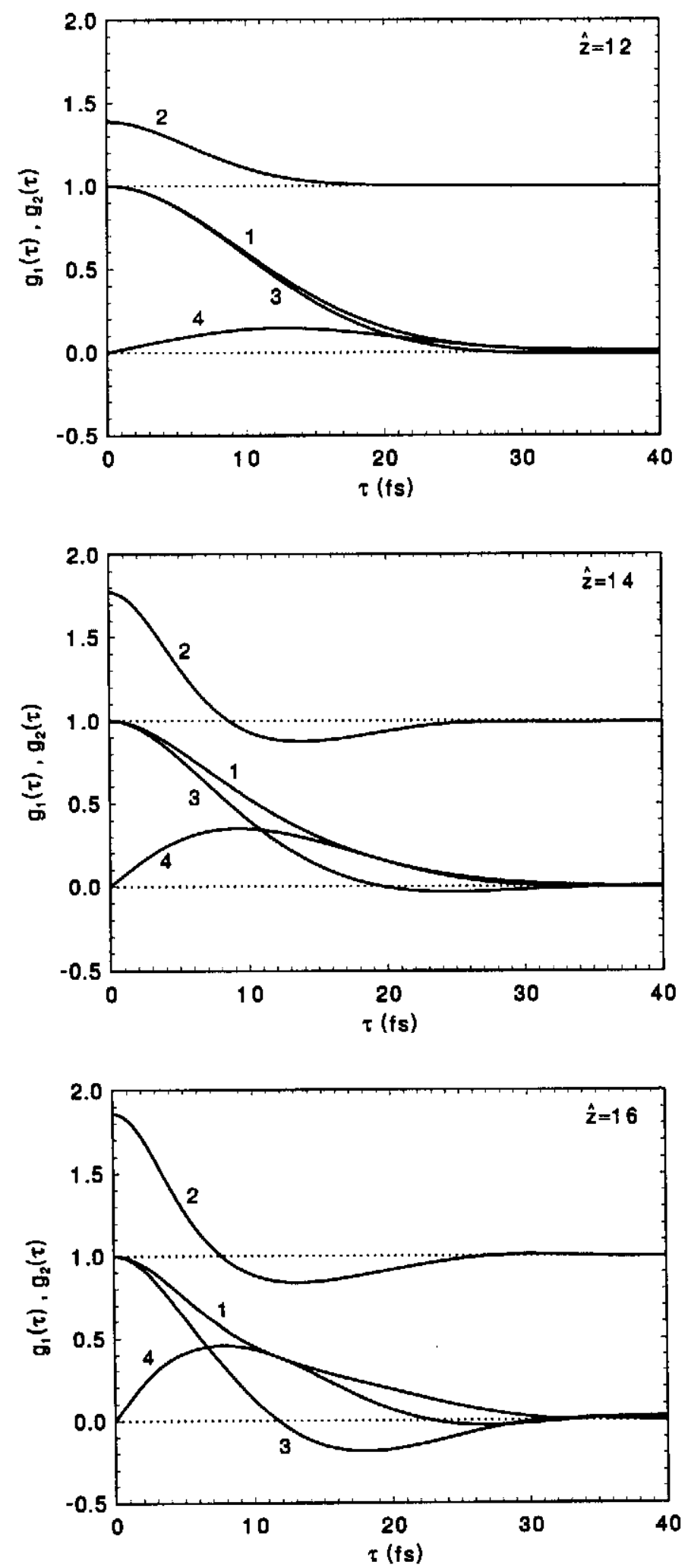

Fig. 5.21. The first and the second order time correlation functions, $\left|g_{1}(\tau)\right|$ (curve 1), $g_{2}(\tau)$ (curve 2), $\operatorname{Re}\left(g_{1}(\tau)\right.$ ) (curve 3 ) and $\operatorname{Im}\left(g_{1}(\tau)\right)$ (curve 4) of the radiation pulse at different reduced length of the FEL amplifier operating in nonlinear regime. Calculations have been performed with nonlinear simulation code over $10^{4}$ independent statistical events. 

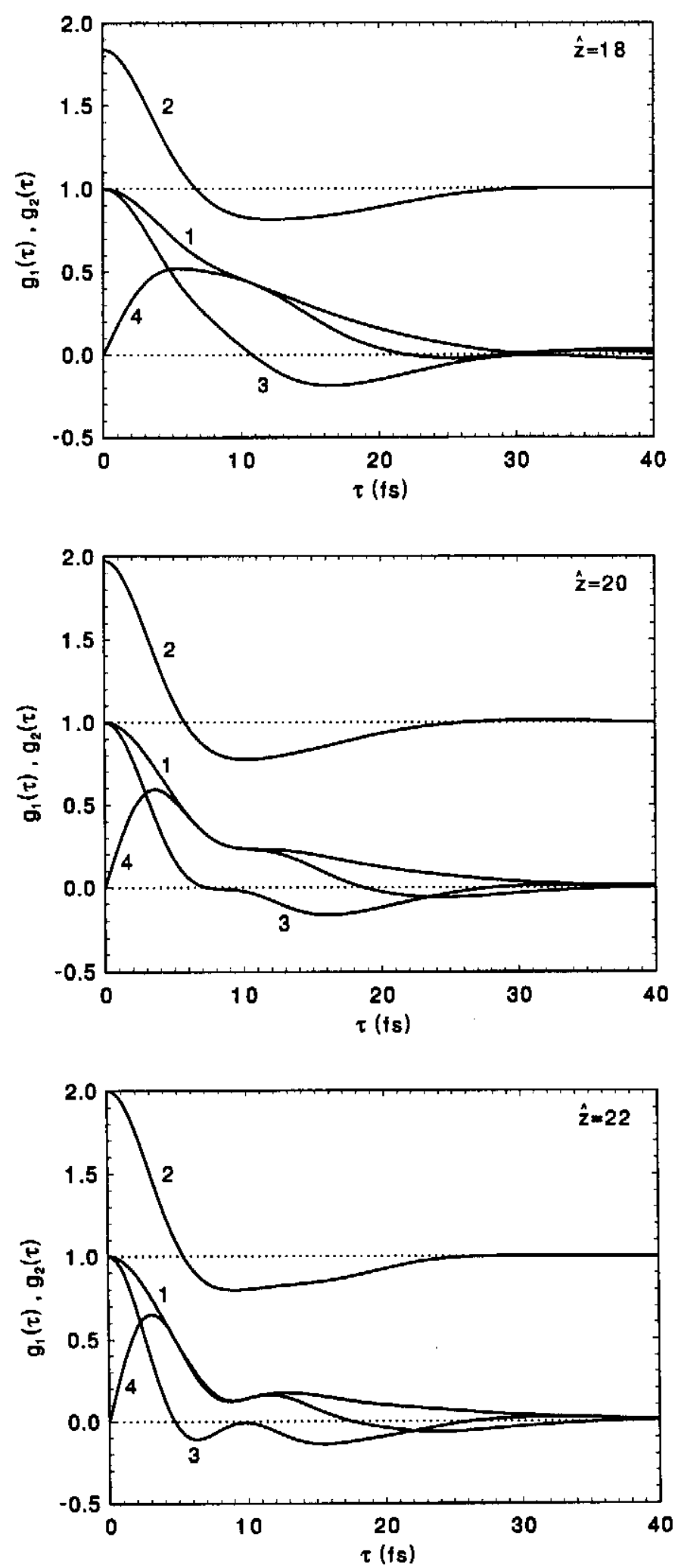

Fig. 5.22. The first and the second order time correlation functions, $\left|g_{1}(\tau)\right|$ (curve 1), $g_{2}(\tau)$ (curve 2), $\operatorname{Re}\left(g_{1}(\tau)\right)$ (curve 3 ) and $\operatorname{Im}\left(g_{1}(\tau)\right)$ (curve 4 ) of the radiation pulse at different reduced length of the FEL amplifier operating in nonlinear regime. Calculations have been performed with nonlinear simulation code over $10^{4}$ independent statistical events. 


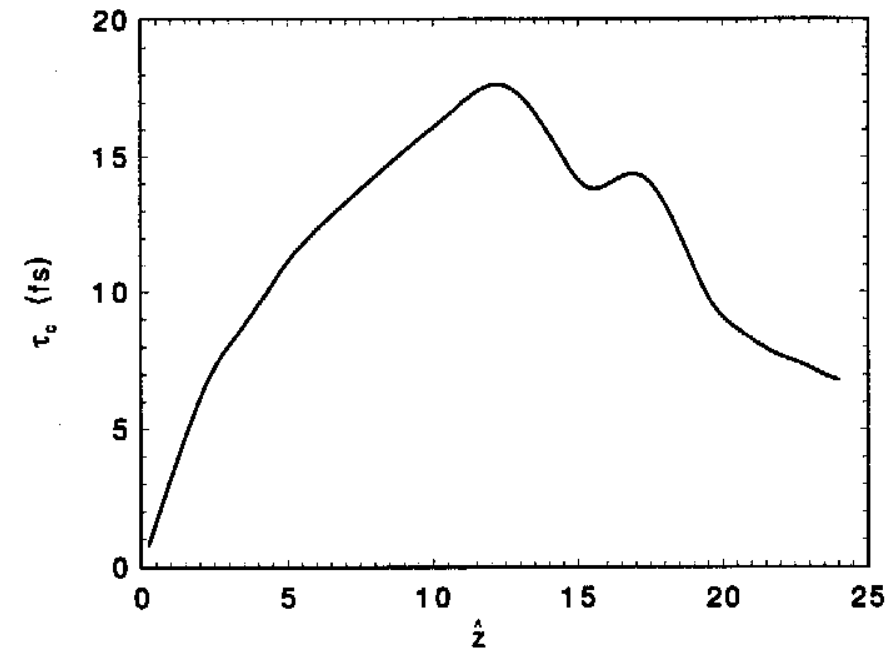

Fig. 5.23. Coherence time $\tau_{\mathrm{c}}$ as function of the reduced length of the FEL amplifier. The values of $\tau_{\mathrm{c}}$ have been calculated by means of integration of the first order time correlation function (see Figs. 5.15, 5.21 and 5.22 , and eq. (2.52)). Calculations have been performed with nonlinear simulation code.

Coherence time, $\tau_{c}$ (see eq. (2.52), decreases drastically in the nonlinear regime (see Fig. 5.23).

The spectrum of SASE FEL. In Figs. 5.24 and 5.25 we present the evolution of the radiation spectrum in the nonlinear mode of operation. Spectrums have been reconstructed using calculated values of the first order time correlation function (see Figs. 5.21 and $5.22)$.

The first and the second order spectral correlation functions. In Fig. 5.26 we present the first and the second order spectral correlation functions for the radiation of SASE FEL operating at saturation. With the accuracy of performed calculation we can state that spectral correlation functions are the same as those for the linear mode of the SASE FEL operation (see Fig. 5.7).

Fluctuations of the radiation power. In Fig. 5.27 we present the results of calculation of the normalized rms deviation of the instant fluctuations of the radiation power, $\sigma_{\mathrm{p}}=$ $\left\langle(P-\langle P\rangle)^{2}\right\rangle^{1 / 2} /\langle P\rangle$, as function of the undulator length. One can see from this figure that at the linear stage of the SASE FEL operation the value of the deviation is equal to unity. This case has been analyzed in detail in the section devoted to the linear theory. It has been shown that the probability density distribution is described by the negative exponential law. In the nonlinear mode of operation the deviation of the power fluctuations differs significantly from unity. It indicates that the probability density function should differ from the negative exponential law. Figs. 5.28 - 5.30 present the distributions of the instant power fluctuations at different length of the undulator. It is interesting to notice that in the deep nonlinear regime this distribution tends again to the negative exponential one and the value of the rms deviation also tends to unity (see Fig. 5.27). 

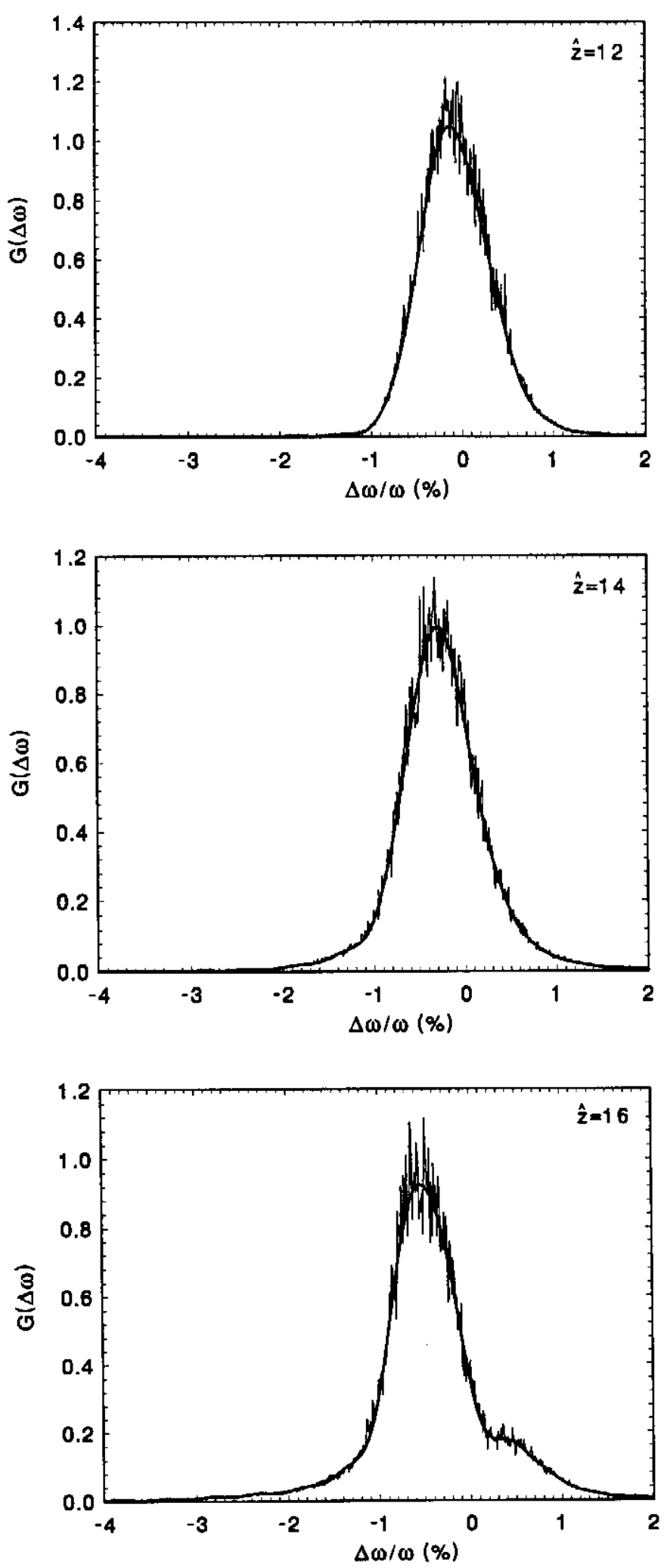

Fig. 5.24. Normalized spectrum of the radiation from SASE FEL $G(\Delta \omega)$ at different reduced length of the FEL amplifier operating in nonlinear regime. Calculations have been performed with nonlinear simulation code over 100 shots. Smooth curves present the spectrum reconstructed from the first order time correlation function (see Fig. 5.21 and eq. (5.1)). 

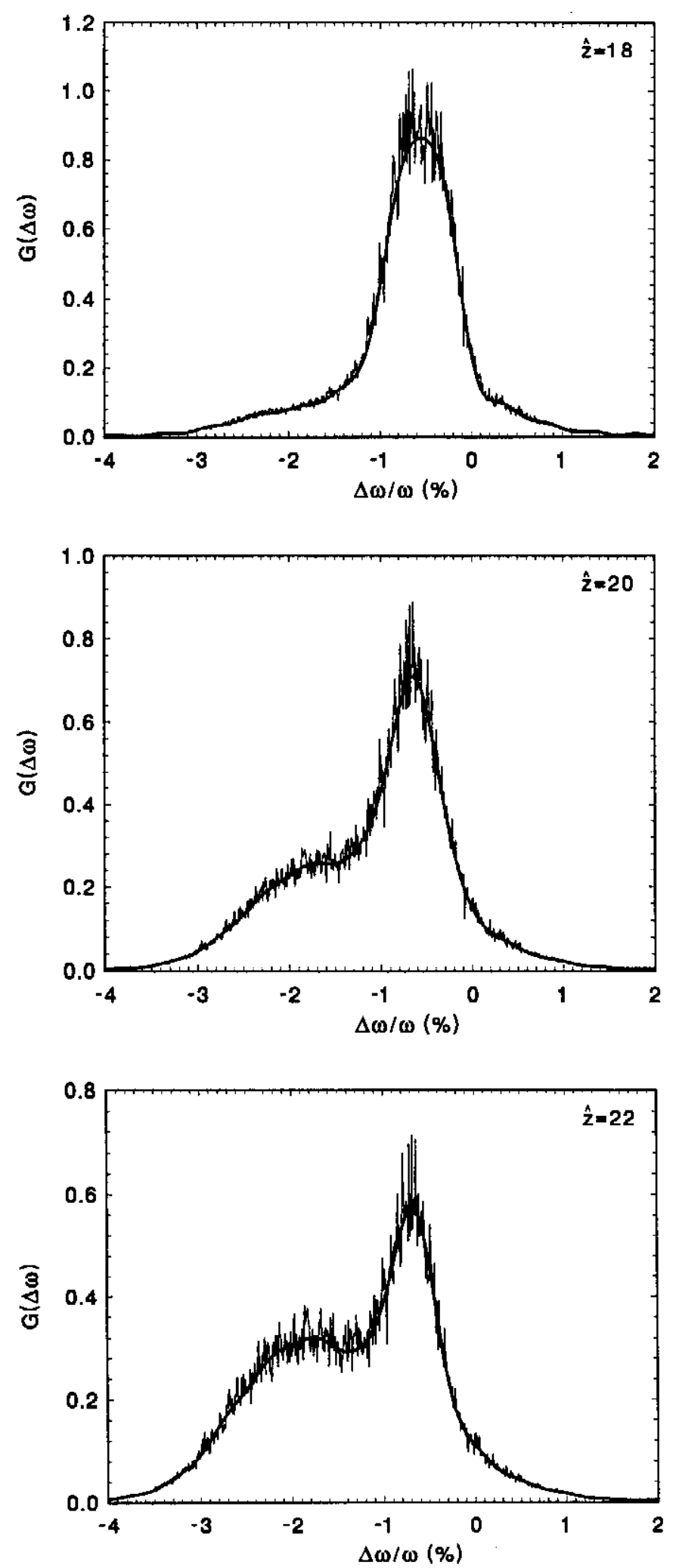

Fig. 5.25. Normalized spectrum of the radiation from SASE FEL $G(\Delta \omega)$ at different reduced length of the FEL amplifier operating in nonlinear regime. Calculations have been performed with nonlinear simulation code over 100 shots. Smooth curves present the spectrum reconstructed from the first order time correlation function (see Fig. 5.22 and eq. (5.1)). 

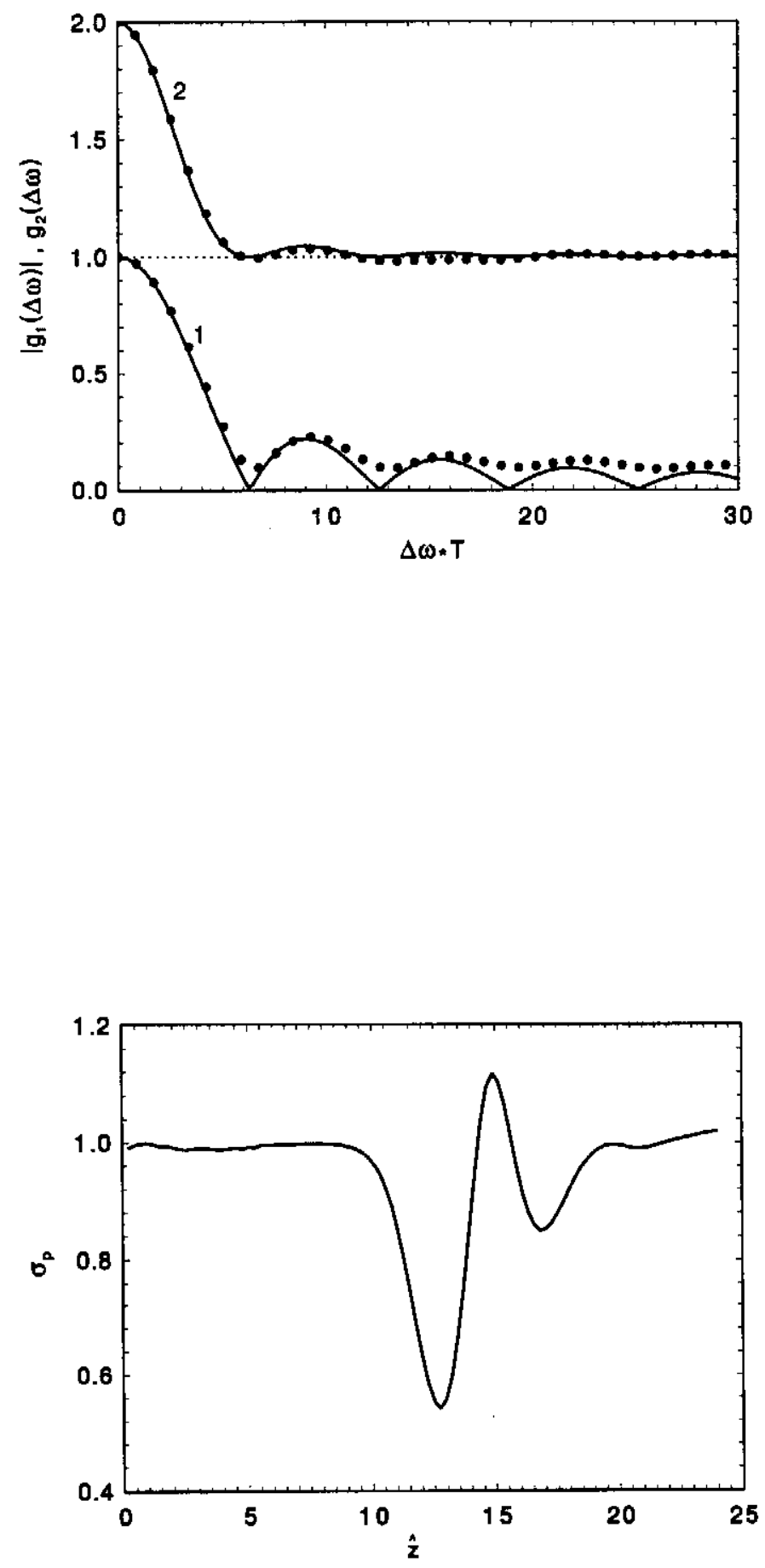

Fig. 5.26. The module of the first order spectral correlation function, $\left|g_{1}(\Delta \omega)\right|$ (curve1), and the second order correlation function, $g_{2}(\Delta \omega)$ (curve 2), of the radiation pulse at the reduced length of the FEL amplifier $\hat{z}=13$ (saturation point). Solid curves are the results of calculations with analytical formulae (2.26) and (2.31). Circles are the result of calculations with nonlinear simulation code over 500 independent statistical events.

Fig. 5.27. Normalized rms deviation of the instant fluctuations of the radiation power, $\sigma_{p}$, as function of the reduced length of the FEL amplifier. Calculations have been performed with nonlinear simulation code over $10^{4}$ independent statistical events. 

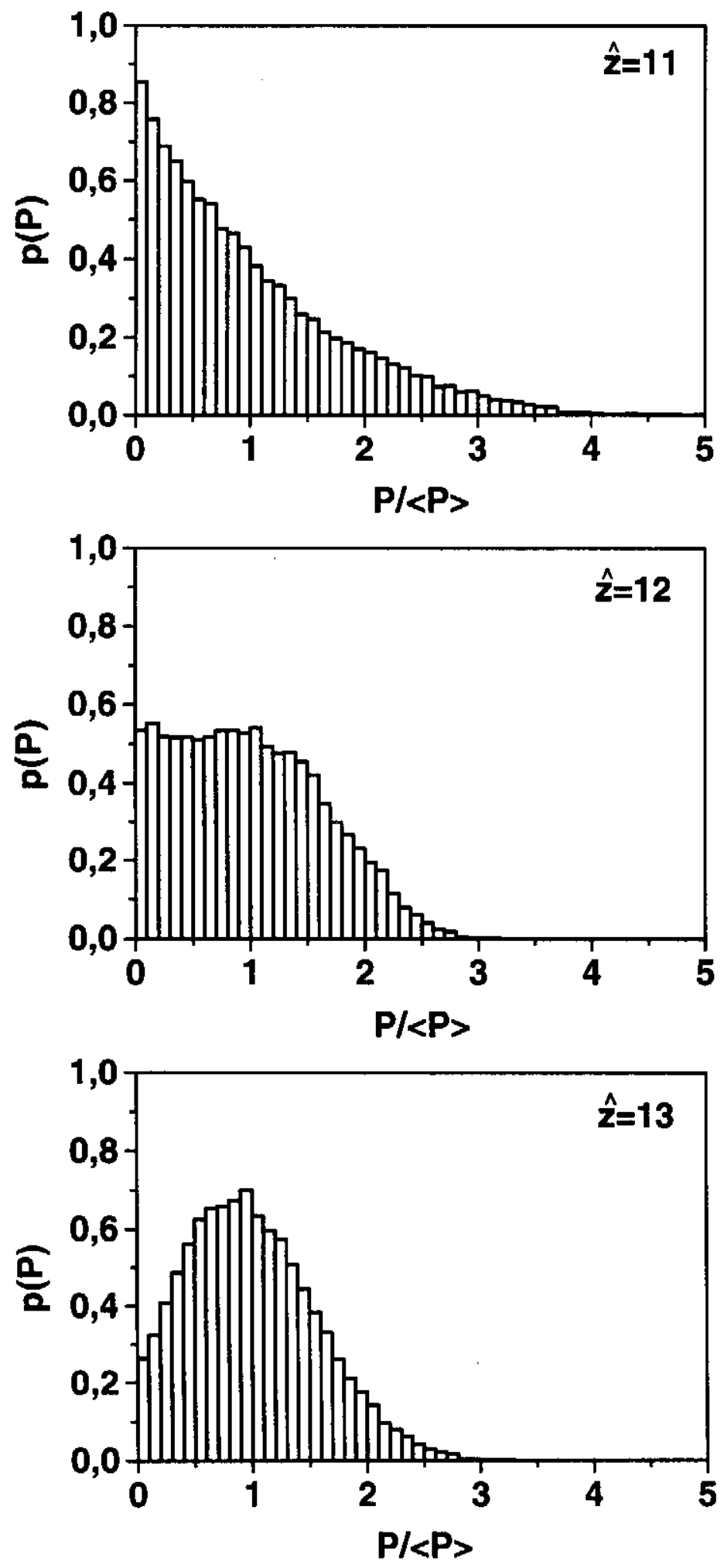

Fig. 5.28. Histograms of the probability density distribution, $p(P)$, of the instant output power at different reduced length of the FEL amplifier. Calculations have been performed with nonlinear simulation code over $10^{4}$ independent statistical events. $\langle P\rangle$ denotes the average power. 

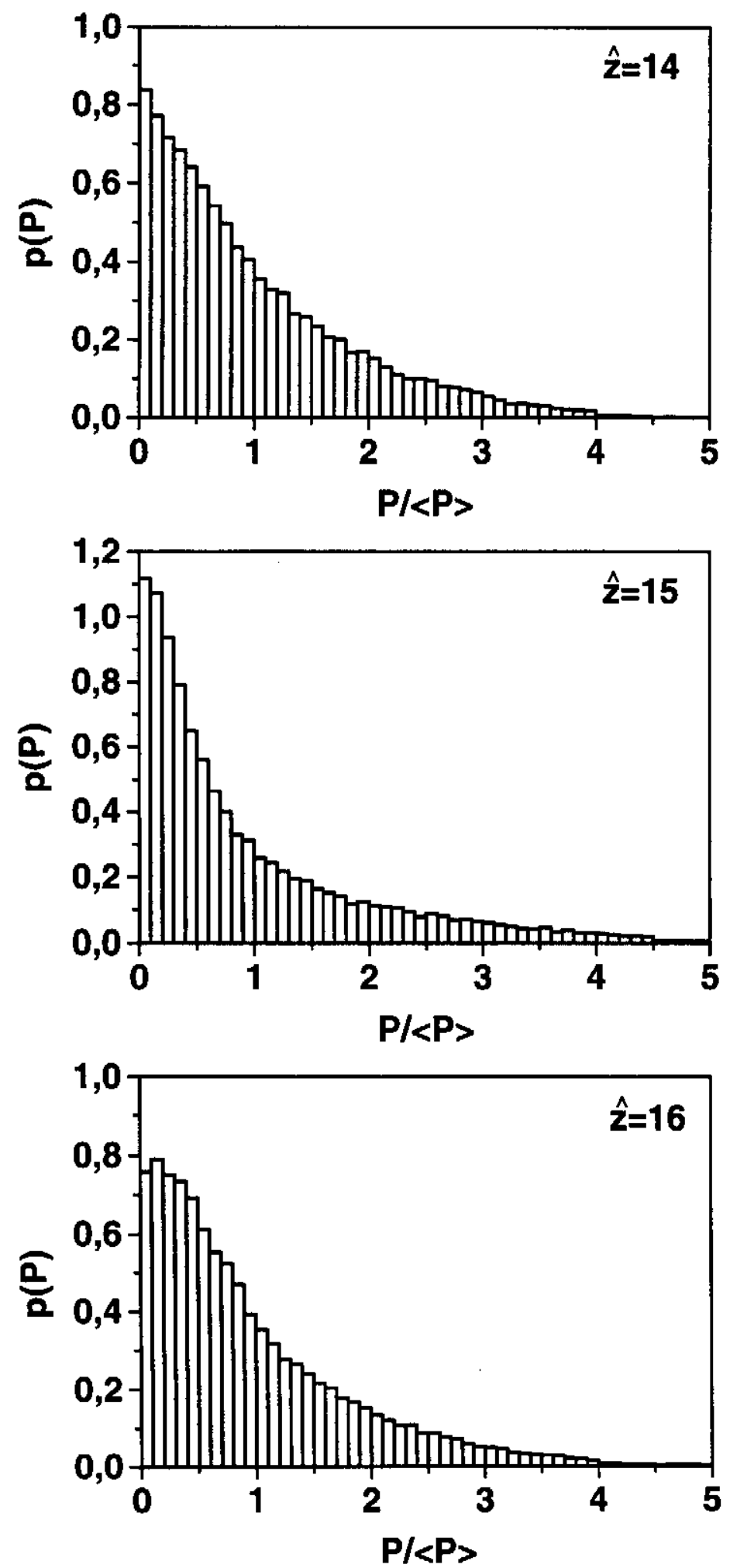

Fig. 5.29. Histograms of the probability density distribution, $p(P)$, of the instant output power at different reduced length of the FEL amplifier. Calculations have been performed with nonlinear simulation code over $10^{4}$ independent statistical events. $\langle P\rangle$ denotes the average power. 

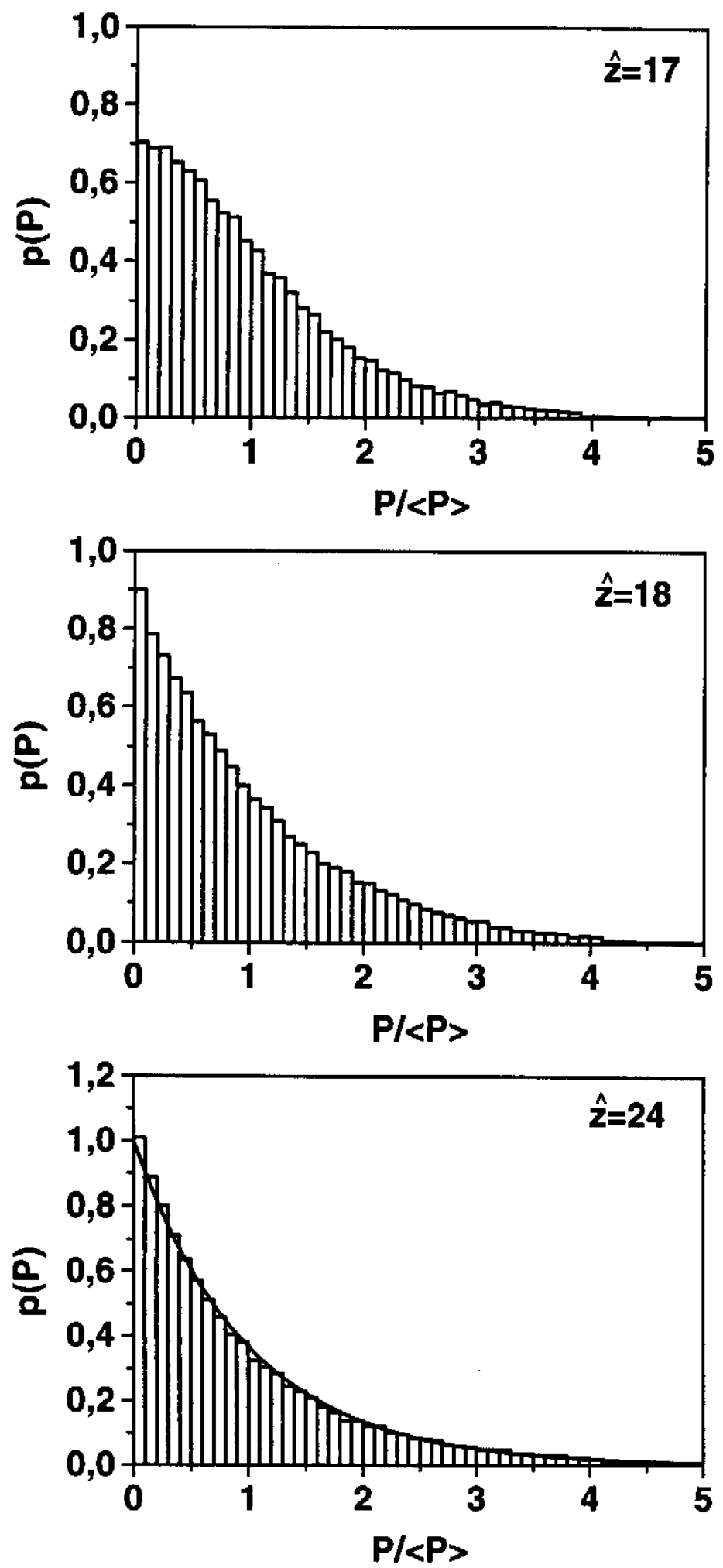

Fig. 5.30. Histograms of the probability density distribution, $p(P)$, of the instant output power at different reduced length of the FEL amplifier. Calculations have been performed with nonlinear simulation code over $10^{4}$ independent statistical events. $\langle P\rangle$ denotes the average power. Solid curve represents the negative exponential distribution $p(P)=$ $\exp (-P /\langle P\rangle)$. 
Fluctuations of the energy in the radiation pulse. We performed numerical study of the fluctuations of the energy in the radiation pulse integrated over finite time. Fig. 5.31 presents the normalized rms deviation of the energy fluctuations in the radiation pulse as function of the undulator length. It is seen that the fluctuations achieve their maximum in the end of linear regime. The first local minimum corresponds to the saturation point $(\hat{z}=13)$. This is in a good agreement with Figs. 5.27 and 5.28 showing that relative fluctuations of the instant radiation power achieve their minimum at the saturation point.
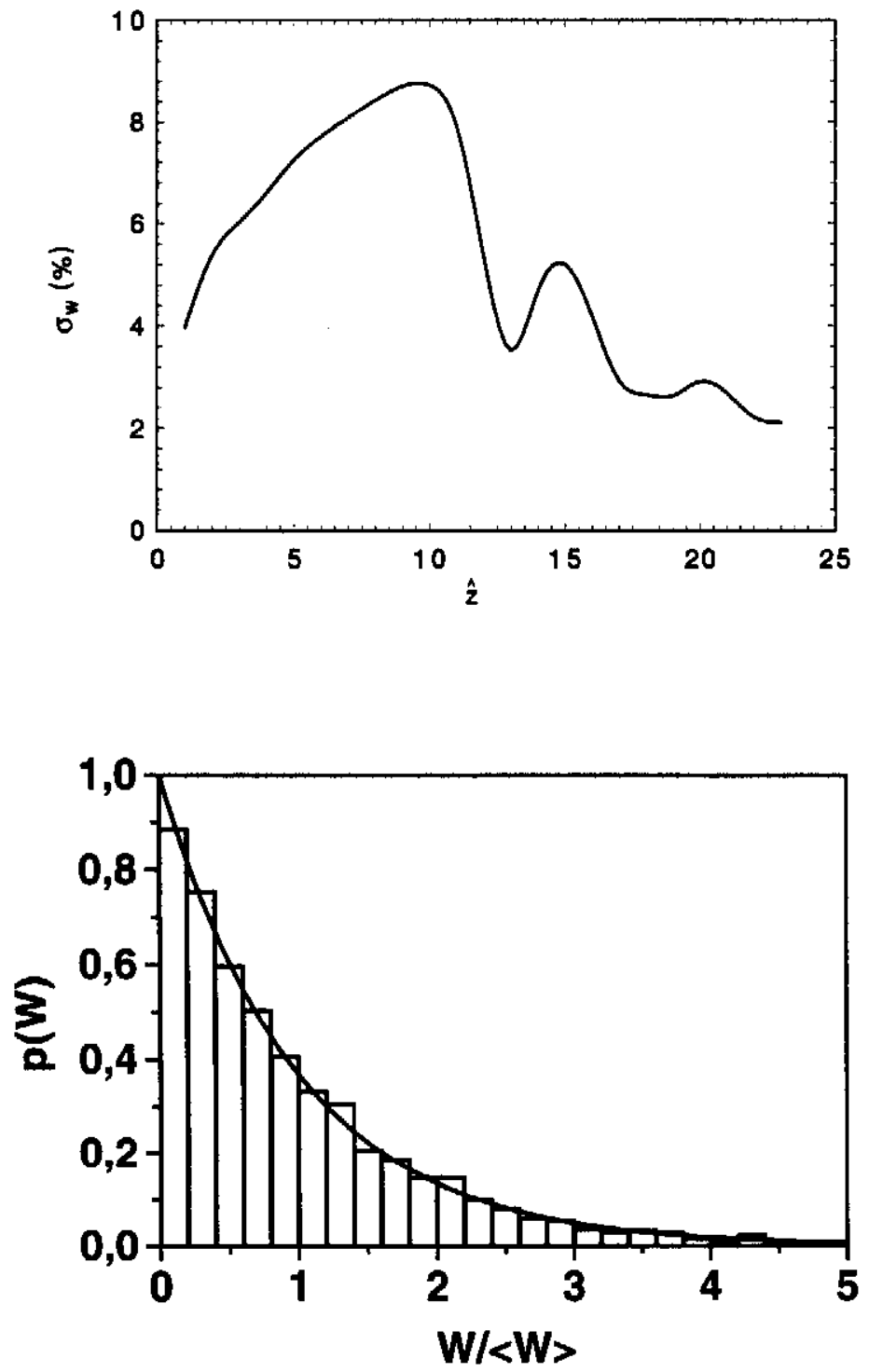

Fig. 5.31. Normalized deviation of the energy fluctuations in the radiation pulse, $\sigma_{\mathrm{W}}$, as function of the reduced length of the FEL amplifier. Calculations have been performed with nonlinear simulation code over 100 shots.

Fig. 5.32. A histogram of the probability density distribution, $p(W)$, of the radiation energy after monochromator. The reduced length of the undulator is equal to $\hat{z}=13$ (saturation point). The width of the monochromator is less than characteristic width of the spikes in the spectrum (see Fig. 5.19). Calculations have been performed with nonlinear simulation code over $10^{3}$ independent statistical events. $\langle W\rangle$ denotes the average energy. The solid curve represents the negative exponential distribution $p(W)=\exp (-W /\langle W\rangle)$.

Fluctuations of the radiation energy at the exit of monochromator. In Fig. 5.32 we present the histogram of probability density distribution of the radiation energy after the monochromator. The reduced length of the undulator is equal to $\hat{z}=13$ (saturation point). The width of the monochromator is less than characteristic width of the spikes in 
the spectrum. It is seen from this plot that at a small window of the monochromator the fluctuations of the radiation energy follow the negative exponential law.

\section{Acknowledgement}

We wish to thank J. Feldhaus, P. Gürtler, J. Krzywinski, B. Lengeler, G. Materlik, J. Pflüger, J. Roßbach, J.R. Schneider and B. Sonntag for useful discussions. We are grateful to V. Heynen, H. Schultz and S. Wipf for organization of technical support for computer simulations. 
TESLA FEL-Report 1997-02 


\section{References}

1. A.M. Kondratenko and E.L. Saldin, Part. Acc. 10(1980)207.

2. Ya.S. Derbenev, A.M. Kondratenko and E.L. Saldin, Nucl. Instrum. and Methods 193(1982)415.

3. R. Bonifacio, C. Pellegrini and L. Narducci, Opt. Commun. 50(1984)373.

4. J.B. Murphy and C. Pellegrini, Nucl. Instrum. and Methods A237(1985)159.

5. R. Tatchyn et al., Nucl. Instrum. and Methods A375(1996)274.

6. Linear Collider Conceptual Design Report, DESY print, April 1997.

7. "A VUV Free Electron Laser at the TESLA Test Facility: Conceptual Design Report", DESY Print TESLAFEL 95-03, Hamburg, DESY, 1995.

8. J. Rossbach, Nucl. Instrum. and Methods A375(1996)269.

9. W. Brefeld et al., "Parameter Study on Phase I of the VUV FEL at the TESLA Test Facility", DESY Print TESLA-FEL 96-13, to be published in Nucl. Instrum. and Methods (Proc. of 19th FEL Conf.).

10. Proc. of Workshop on Scientific Applications of Coherent X-rays, (Stanford, February 1994), preprint SLAC437.

11. R. Bonifacio and F. Casagrande, Nucl. Instrum. and Methods A237(1985)168.

12. K.J. Kim, Nucl. Instrum, and Methods A250(1986)396.

13. K.J. Kim, Phys. Rev. Lett. 57(1986)1871.

14. J.M. Wang and L.H. Yu, Nucl. Instrum. and Methods A250(1986)484.

15. S. Krinsky and L.H. Yu, Phys. Rev. A35(1987)3406.

16. R. Bonifacio et al., Phys. Rev. Lett. 73(1994)70.

17. P. Pierini and W. Fawley, Nucl. Instrum. and Methods A375(1996).

18. E.L. Saldin, E.A. Schneidmiller and M.V. Yurkov, "Simulation Studies of $6 \mathrm{~nm}$ Free Electron Laser at the TESLA Test Facility Starting from Noise", DESY print TESLA-FEL 96-07.

19. J. Goodman, Statistical Optics, (John Wiley and Sons, New York, 1985).

20. R. Bonifacio et al., Riv. Nuovo Cimento, Vol.13, No.9 (1990).

21. E.L. Saldin, E.A. Schneidmiller and M.V. Yurkov, Phys. Rep. 260(1995)187.

22. M. Abramowitz and I.A. Stegun, Handbook of Mathematical Functions, (National Bureau of Standards, 1964).

23. H. Wiedemann, Particle Accelerator Physics II, (Springer-Verlag, Berlin-Heidelberg,1995).

24. L. Mandel, Proc. Phys. Soc. (London), 1959, v.74, p.223.

25. C. Penman and B.W.J. McNeil, Opt. Commun. 90(1992)82.

26. J. Krzywinski, E.L. Saldin, E.A. Schneidmiller and M.V. Yurkov, DESY print TESLA-FEL 97-03. 